\title{
Philosophiques
}

\section{Le trop dit, le dit, le mal dit et le non dit dans le discours althusserien sur l'idéologie}

\section{Danièle Letocha}

Volume 9, numéro 1, avril 1982

URI : https://id.erudit.org/iderudit/203183ar

DOI : https://doi.org/10.7202/203183ar

Aller au sommaire du numéro

Éditeur(s)

Société de philosophie du Québec

ISSN

0316-2923 (imprimé)

1492-1391 (numérique)

Découvrir la revue

Citer cet article

Letocha, D. (1982). Le trop dit, le dit, le mal dit et le non dit dans le discours althusserien sur l'idéologie. Philosophiques, 9(1), 41-94.

https://doi.org/10.7202/203183ar
Résumé de l'article

Ce texte vise à établir, au-delà des excès de la mode, le bilan des apports althussériens à la théorie des idéologies. Nous opposons d'abord le marxisme épistémologique à ses propres exigences de scientificité. Le bilan est négatif quant au recours à la théorie, au matérialisme, à la dialectique et à la structure. Nous proposons de résoudre certains des problèmes en substituant la dichotomie culture/idéologie à l'opposition althussérienne science/idéologie. 


\title{
LE TROP DIT, LE DIT, LE MAL DIT ET LE NON DIT DANS LE DISCOURS ALTHUSSERIEN SUR L'IDÉOLOGIE
}

\author{
par Danièle Letocha
}

\begin{abstract}
RÉSUMÉ. Ce texte vise à établir, au-delà des excès de la mode, le bilan des apports althussériens à la théorie des idéologies. Nous opposons d'abord le marxisme épistémologique à ses propres exigences de scientificité. Le bilan est négatif quant au recours à la théorie, au matérialisme, à la dialectique et à la structure. Nous proposons de résoudre certains des problèmes en substituant la dichotomie culture/idéologie à l'opposition althussérienne science/idéologie.
\end{abstract}

ABSTRACT. This paper presents a North American criticism of a Parisian variety of marxism which " made a hit " during the sixties : Louis Althusser's epistomological marxism. It does not yield what it promised, mainly a scientific philosophy. It is analysed here on a specific point : the theory of ideology. In our view, Althusser fails to define science as the rational counterpart of ideology.

" Ma malignité s'en allait ; je me plaisais à ce cas-là, après tant d'autres. J'admets tout. Si je condamnais les hommes, les connaîtrais-je?"

Drieu La Rochelle Le lieutenant de tirailleurs.

Comment circonscrire en quelques pages les ravages d'une mode? Les malheurs qui recouvrent la personne privée de Louis Althusser imposent-ils un moratoire pudique sur toute discussion concernant son enseignement et ses écrits? Je ne le crois pas. Pourquoi ajouter le poids d'un silence embarrassé à la désaffection passive qui touchait déjà la doctrine althusérienne depuis quelques années? Ce vide laisse libre cours à l'opportunisme paresseux et bavard de la prétendue nouvelle droite. Les héros parisiens sont fatigués, semble-t-il. 
En effet, l'âge d'or d'un certain type de gauche paraît aujourd'hui définitivement révolu. Il aura duré environ quinze ans. Plutôt que de s'en détourner, la critique peut empêcher qu'on évacue le bébé avec l'eau de la baignoire, comme disent les Américains. Quoiqu'il y ait un peu du charognard chez tout philosophe, il ne s'agit pas de s'acharner sur un vaincu, mais plutôt d'opérer une mise en perspective. Maintenant que la poussière retombe sur le chemin, comment faire la part du feu et distinguer le programme de la doctrine, les concepts des méthodes, les échecs des acquis ? Peut-être une telle démarche sera'-t-elle le fait de quelques penseurs situés à la "périphérie " chère à Althusser. Ici, du moins, ceux qui se montrèrent rétifs à l'engouement hexagonal refusent, pour cette raison même, d'en porter le deuil. Dès 1973, en effet, au sommet de l'arrogance des althussériens, des objections précises leur étaient faites. Pour en nommer quelques-unes: celles de Maurice Lagueux (in Culture et société, 1973), de Claude Savary (au congrès de Varna, 1973) et de Fernand Dumont (Les idéologies, 1974). Le débat sur le fond ne put s'engager, l'adversaire étant assourdi par les cris enthousiastes de ses disciples. Le bilan servile d'un Saül Karsz (Thóorie et politique : Louis Althusser, 1974) ne pourra pourtant servir d'apologie finale aux yeux de personne. Or, le climat s'étant assaini, il devient possible de rouvrir le dossier: que les interprètes soient marxistes ou non ne sera sans doute pas décisif, pourvu qu'ils pensent au lieu de prêcher. C'était là trop demander, il y a seulement dix ans, alors que Raymond Aron se résignait mal à poursuivre "un dialogue sans espoir et sans issue " (D'une sainte famille à l'autre, 1969, dont les deux cents pages sur Althusser ne sont pas retenues dans la bibliographie partiale de Karsz).

Ces quelques pages ne marquent évidemment pas l'envol de la chouette au soir des épisodes dramatiques du devenir de la conscience de soi. Elles visent plus modestement à dépouiller la thématique originelle d'Althusser de quelques oripeaux qui ont lancé la vogue de l'althussérisme dans les coteries universitaires et journalistiques au début des années soixante. Il a paru intéressant de s'interroger sur les rapports entre l'habit et le moine pour voir dans quelle mesure les textes 
théoriques d'Althusser se prêtent effectivement à ces extrapolations et à ces simplifications. La définition scientifique de l'idéologie, thèse centrale, sera abordée sous les angles suivants : l'image de l'opposition vérité/erreur dont elle est tributaire ainsi que l'insuffisance du travail fondationnel et analytique qui empêche la notion d'idéologie d'accéder au statut de concept. Première constatation épistémologique : en dépit du "théoricisme " qu'on lui a reproché, Althusser n'a pas suivi le programme de scientificité qu'il s'était lui-même fixé. Seconde constatation : cet échec a hypothéqué l'entreprise de clarification des superstructures, dans le sens annoncé par ses prolégomènes. L'opposition fameuse entre science et idéologie constitue, à nos yeux, un obstacle épistémologique dans le sens suivant: elle rend la théorie aveugle aux phénomènes appartenant au registre symbolique du donné, de l'a priori. Rapportant tout élément à ses conditions de production, ce discours éminemment réducteur reste, en première comme en dernière instance, unidimensionnel. Il devient discours-problème par sa prétention à l'exhaustivité et à l'universalité, en quoi il ne fait que reproduire Marx, sans les excuses historiques de ce dernier. On montrera comment le recours à la structure, comme discours secondaire, isole l'instance idéologique dans un modèle inconciliable avec le marxisme. On voudrait ensuite ouvrir quelques avenues barrées par le "généticisme » althussérien. En effet, l'ordre symbolique a ses raisons que la raison dialectique ne connaît point. Et dans une perspective élargie, il apparaît que le modèle d'Althusser peut efficacement rendre compte de certaines configurations conflictuelles de l'objet-société tandis que l'objet-culture y échappe. On tentera d'établir que les sciences de la culture sont fécondes dans la mesure où elles ont recours au modèle analytique.

Est-il bien utile de revenir aujourd'hui sur des thèmes tellement ratissés qu'ils en sont devenus des poncifs? Qu'en est-il des questions adressées à ces thèses depuis quinze ans, tant sur le plan externe qu'interne? Au pire, elles ont été ignorées ; au mieux, anathématisées. Car c'est le propre de la mode de marquer les bornes de son exclusive par des mouvements d'humeur plutôt que par des arguments. 
Commençons donc par traverser ce brouillard séducteur. On voudrait contribuer à le dissiper entièrement pour qu'au delà de ces propos, le débat passe enfin du poncif à la question, de la thèse à l'hypothèse.

\section{LA GAUCHE TRIOMPHANTE : LA TACTIQUE DU DISCOURS ET LE DISCOURS À LA MODE}

L'événement peut se situer entre deux suicides : celui de Sebag, en 1965, et celui de Poulantzas, en 1979. Bien que le maître ait cessé d'enseigner dès 1975, l'aura s'est prolongée par une sorte de « rétention académique " (analogue à la rétention rétinienne). Conformément aux lois du décalage, le sommet d'intensité polémique se repère entre 1969 et 1972.

C'est l'audience quasi immédiate des premiers articles du maître qui est remarquable. Comme Althusser l'a noté, les théoriciens du marxisme sont rares dans la tradition française. Grâce à lui, le début des années soixante (période de prospérité économique, ouverte par les accords d'Évian) voit la rencontre d'un projet théorique avec des volontés, des convictions et des engagements pratiques, lesquels croient trouver, dans cette quête du Graal, la légitimité et le prestige universitaire (et non des moindres) garantissant leur action politique. D'où un mouvement d'identification et de ralliement des intellectuels marxistes parisiens d'abord, français ensuite, enchantés de se débarrasser des déchirements réactionnaires des Sartre, Garaudy ou Lefebvre. Car chez Althusser, la théorie se donne comme " praxis théorique»; la science est une pratique différentielle à caractère théorique. Subordonné à la lutte des classes, ce discours se présente explicitement comme un instrument au service du prolétariat et plus précisément de son avant-garde : le parti communiste français. Comme l'a noté Althusser lui-même, c'est là une formule qui a permis aux « intellectuels de gauche" de dédouaner leur conscience malheureuse : les uns se consolant de n'être pas nés ouvriers, les autres d'être fils d'ouvriers sans avoir à trahir. Cette réconciliation superficielle n'a duré qu'un temps. La crise de mai 68 fit resurgir plus tard les démons du soupçon. Les tanks soviétiques, entrant à Prague quelques mois après, produisirent moins de tensions internes, ce qui reste significatif de l'ethnocentrisme hexago- 
nal. Néanmoins, au début des années soixante, les positions d'Althusser suscitent une rare unanimité que la mode institue trop rapidement en discours du marxisme dominant. En effet, les premiers articles d'Althusser, qui se présentaient comme lieux d'interrogation et jalons d'une démarche à peine initiée, servent aussitôt de normes d'allégeance à la seule ligne correcte et rédemptrice. Une même assurance cinglante envahit tous les adeptes. Le mécanisme du cénacle rassemble et clôt d'un même mouvement : il faut se prononcer pour ou contre le prophète. Ainsi, des notions à peine ébauchées (et souvent prometteuses) se figent et son invoquées comme catégories définitives par les zélateurs impatients : science, champ idéologique, appareils idéologiques d'État (réduits au sigle AIE pour les familiers), autonomie relative, coupure épistémologique, surdétermination, instance, symptôme, etc., sont élevés à la dignité de « concepts critiques».

L'alignement sur l'orthodoxie fait barrage à la considération des postulats et des limites de la doctrine. On passe aux applications, rigides, multiples et souvent hâtives, d'autant plus dogmatiques que leur fondement est incertain. On ne s'embarrasse guère de procédures de vérification/falsification du modèle théorique. Les seules objections tolérées portent sur des nuances poliment suggérées, à usage interne: par exemple, Poulantzas se demande si Bourdieu n'infléchirait pas un peu trop le concept de classe vers celui, plus mécanique, de caste.

Les grands disciples sont nombreux dans cette première phase. Pour identifier quelques voix dans ce courant, citons en sociologie : Bourdieu, Passeron, Darbel, Baudelot, Establet ; en épistémologie : Balibar, Badiou, Lecourt ; en politicologie : Poulantzas, Rancière (jusqu'à la rupture de 1968). Les compétences scientifiques qu'Althusser juge pourtant fondamentales (« Problèmes étudiants », 1964) n'empêchent pas les uns de se mêler des affaires des autres : tous sont historiens socio-politicologues. Mais aucun n'est économiste. Voilà des marxistes qu'on ne peut taxer d'économisme. Entre eux, peu de confrontations théoriques. Le faible degré d'appropriation personnelle de la théorie empruntée illustre peut-être le thème de la mort du sujet. Les disciples se situent dans une mouvance que manifestent partout la même rhétorique cassante et les 
mêmes précautions pour parler du maître. Plus encore, ce texte à plusieurs voix frappe le lecteur simplement curieux par l'extrême transférabilité de l'appareil théorique, sans égard à la spécificité des champs et des objets. Tout passe dans la moulinette des conditions de production du capitalisme avancé : les données qui s'aviseraient de grincer sont rejetées dans la géhenne de l'imaginaire, de l'illusoire, bref de l'irréel car la machine ne s'enraye jamais.

Grégarisme et complaisance intellectuels cimentent la mode de l'althussérisme à laquelle le maître ne trouve rien à redire (par la plume, du moins). Convenons-en, si c'est une circonstance atténuante, la pensée française dans son ensemble s'est montrée particulièrement vulnérable à la mode pendant les deux dernières décennies. On a vu le lacanisme, le blanchotisme, le derridisme compter leurs fideles et rompre bruyamment avec tel individu, telle revue savante ou tel magazine. Les étiquettes font fureur. Plus que les autres, l'althussérisme a pratiqué l'excommunication et dénoncé globalement tout adversaire touché par la " contamination » de l'idéalisme bourgeois (dont Marx jusqu'en 1845 !). Cette mode n'aurait pu prospérer sans un réseau institutiontel favorable et l'université française se prête, on le sait, à ces coquetteries. Pour parler du marxisme rue d'Ulm, ou à Nanterre, ou à Vincennes, il était difficile, alors, de ne pas montrer patte blanche. Les Éditions de Minuit accueillent les textes de recherche appliquée tandis que la collection "Théorie", fondée en 1965 par Althusser chez Maspero, publie les travaux historiques et épistémologiques. Le feuillet d'information, inséré dans les exemplaires de cette dernière collection à partir de 1973, nous instruit sur le genre de critique qui intéresse les auteurs : «Quand une critique est sérieuse et honnête, quand elle est le fait d'un lecteur qui essaie lui aussi d'être marxiste, qui essaie lui aussi de devenir communiste, elle rend vraiment service. "Pourtant, on vient de lire plus haut la déclaration suivante : "Car le marxisme n'est pas une religion où il suffit d'adhérer à un petit nombre de dogmes bien présentés, et de répéter la prière quotidienne. "Voilà des gens qui manquent singulièrement d'humour, entre autres choses. 
L'althussérisme, c'est une mode qui s'est prise au sérieux. Son essoufflement s'explique moins par les pressions externes d'une conjoncture défavorable que par une fatigue narcissique: il est retombé dans le sommeil dogmatique du marxisme français que les premiers textes entendaient secouer (« Aujourd'hui "). Cette doctrine a été victime de la mode dont elle avait profité. Qu'on n'y voie pas une donnée accessoire. Pour les contemporains, en effet, le rapport aux constellations théoriques dominantes ne se vit pas dans la même sérénité que la lecture des textes stratifiés dans la distance historique (et par là également obscurcis, mais d'une autre manière). La " réussite " d'une trame théorique est souvent déroutante. Le malaise que produisent ses grands inquisiteurs, ses chantres et ses épigones est d'autant plus inquiétant lorsqu'il indique vaguement une source vive à repérer, laquelle se prêterait ou non à cette dérive vers le dérisoire. Nietzsche, englué dans les gloses hégéliennes, développe une réaction d'allergie permanente à l'hégélianisme, à toute mode et au rapport pédagogique. Mais faut-il être intempestif à tout prix ?

Le climat étouffant dont on tente ici de rendre compte n'est évidemment pas un élément philosophique. Si l'on veut identifier un interlocuteur philosophique potentiel dans ce décor, il faut procéder par objectivation. Pour ramasser un bilan provisoire, on pourrait dire qu'au départ, Althusser pose la science marxiste comme visée totalisante et universaliste, en quoi il est fidèle à Marx. L'althussérisme, en tant que mode, a fait glisser ce discours vers le totalitarisme intellectuel. Au sommet de cette euphorie, nous nous trouvons devant une figure sophistiquée du discours unitaire : celui du parti et de sa ligne "correcte ». C'est là un phénomène socio-littéraire tout à fait distinct de l'esprit qui anime l'école de Francfort, par exemple. Ce sont les attitudes et non les contenus qui caractérisent une mode intellectuelle. Deux pôles rassembleraient les traits de celle-ci : terrorisme et dogmatisme.

Terrorisme d'abord, qui oblitère la question de la validation des discours dans les divers marxismes de l'aprèsguerre. Hors de Cornu, point de salut. Sur le plan des communications externes, tout autre discours est frappé d'interdit et d'aveuglement coupable. Ses questions sont jugées 
irrecevables. C'est l'ère du mépris et du procès d'intention. Par pouvoir déclaratoire, les althussériens disqualifient les adversaires en distribuant les étiquettes infamantes. Ils se disent eux-mêmes liés à une option partisane précise et devant toute objection philosophique, ils invoquent le complot perfide contre leur projet politique. Ils dressent d'ailleurs aimablement leur propre Syllabus dans le feuillet déjà mentionné (et que l'on traite ici comme signe) : «Théorie a ainsi attaqué et démasqué l'Humanisme théorique et l'Économisme (le couple Économisme-Humanisme est la base de toute l'idéologie bourgeoise), l'évolutionnisme, l'historicisme, le positivisme, l'idéalisme juridique, littéraire, ethnologique, scientifique, etc. . .». On remarquera que la liste des thèses hérétiques reste ouverte et que les majuscules distinguent les péchés mortels des véniels. Un lecteur étranger qui se limiterait au corpus althussérien ne pourrait se douter qu'on pensait aussi, en France, hors de ce cercle, car ces textes ne citent pas, ne respirent pas, ne vivent pas. C'est particulièrement frappant d'une pensée qui s'affiche comme dialectique.

Dogmatisme ensuite : ce faisceau de discours qui s'autoalimentent et s'encensent mutuellement a graduellement perdu le sentiment de la résistance du réel (où qu'on le situe) par rapport à l'élaboration spéculative. Par pouvoir postulatoire, l'althussérisme s'est engagé sur la pente stérile d'une certaine intellectualité occidentale. On y décèle facilement la volonté d'assujettir l'univers à la lumière crue de la raison, à l'ordre naif du système, à la transparence rassurante de la scientificité. Depuis que Rousseau a constaté la faillite définitive de toute norme transcendante (pour paraphraser Sebag), le discours français de la modernité se heurte à l'impossibilité de construire une représentation du monde aérée, laïque et qui tolérerait le paradoxe. Seuls quelques esprits « littéraires » tels Diderot ou Valéry ont pu tenir ce pari. De toute évidence, l'althussérisme n'exprime pas cette forme de maturité. Son dogmatisme fait fond sur les écrits marxiens comme sur un roc. On en discute l'exégèse, certes, mais dans un cadre inconditionnel où leur secret, le fameux « noyau rationnel », constitue l'évidence première. Le maître Althusser a lui-même un maître à penser. La question n'est pas de savoir ce qu'il faut lire mais bien de dire comment il faut le lire. Les interventions 
et objections qui enfreignent cette contrainte référentielle perdent toute pertinence pour les althussériens. De plus, le facteur d'adhésion à un parti politique identifié renforce le dogmatisme au niveau des communications internes. L'unanimité est requise des adeptes au nom de la raison pratique : toute dissidence pourrait compromettre la lutte dont la doctrine est un instrument. D'ailleurs, les premières attaques venues de l'intérieur du club sont des règlements de compte à ce niveau : n'étant qu'un révisionniste bourgeois, Althusser se tromperait sur la révolution et la bonne manière de la servir (Rancière, La leçon d'Altbusser, 1974) ; ou encore, le trotskisme de la quatrième Internationale serait la ligne révolutionnaire correcte capable de dénoncer les erreurs marxistes-léninistes (collectif Contre Althusser, 1974). Quiconque a lu ces ouvrages trouvera le ton du présent texte mesuré et même académique... Il reste que l'althussérisme a effectivement joué du chantage à la mauvaise conscience. En se plaçant sous un impératif catégorique, il a enlevé tout intérêt philosophique aux débats de l'époque.

On voit maintenant en quoi le portrait de cette arrogance, aujourd'hui démodée, était nécessaire. La désacralisation du maître, un peu de distance, beaucoup de résistances objectives ont vaincu l'optimisme des jours fastes. Mais nous devons nous rappeler que la rhétorique parisienne a atteint le Québec à retardement, comme il se doit ; son écho pourrait s'y prolonger un peu. C'est surtout l'école, en tant qu'appareil idéologique d'État, qui a fasciné nos althussériens locaux. En 1973, Claude Escande nous donnait, dans Les classes sociales au cegep, une dissertation orthodoxe, bourrée de Bourdieu et cie, chargée de tableaux quantitatifs, mais complètement dépourvue de réflexion critique sur les concepts empruntés et sur leur pertinence. Un degré au-dessous, les émules des émules s'agitent encore. En effet, les récents documents issus des centrales syndicales d'enseignants que sont la F.N.E.Q. C.S.N. et la C.E.Q. reflètent encore la lumière d'astres éteints. Ainsi Les enjeux d'un livre orange (1979) et la critique publiée l'hiver dernier par la C.E.Q., portant sur le document ministériel Un projet éducatif, répètent purement et simplement les généralités moralisatrices de L'école au service de la classe 
dominante et de École et luttes de classes au Québec (1974). En 77, la F.N.E.Q. en donnait un décalque dans L'école publique mise en question. On n'y trouve aucune analyse, aucune proposition opératoire, aucune définition de la spécificité des institutions scolaires dans la société québécoise. Ce sont des manifestes populistes qui appellent à la révolution en empruntant des slogans importés. On a les althussériens qu'on peut. . . Et que dire du recours à Althusser dans l'écriture tourmentée de Bernard Labrousse (L'idéologie dominée, 1978) ? Il est étrange que son projet, intéressant en soi, de faire droit, par une articulation théorique, aux expressions d'une «spécificité culturelle étouffée " s'écrive en contrepoint de la doctrine althussérienne. Le bon élève manifeste une déférence constante au maître qu'il faudrait excuser de n'avoir pas assez pressenti, assez senti, assez saisi le rôle positif de l'enracinement culturel. Que diable Labrousse allait-il faire dans cette galère ? De fait, le modèle althussérien oblitère tout souci culturel. Pour trouver un écho à ce type de préoccupation dans la tradition marxiste, il eût fallu s'adresser à Rosa Luxembourg ou à Gramsci. Mais il est encore plus efficace de lire Fernand Dumont. De toute manière, même ici, les jours maigres sont arrivés et il faut s'en réjouir. Il devient enfin possible d'interroger une entreprise significative en lui rendant ses proportions et en la situant, parmi d'autres, sur le terrain philosophique de la recherche d'une intelligibilité.

\section{PAR DELÀ LE BRUIT : L'INTÉRÊT PHILOSOPHIQUE D'UNE RELECTURE ET D'UNE LECTURE}

Il faut maintenant tenter de prendre la mesure de la contribution apportée par la théorie d'Althusser à l'histoire du marxisme et à l'épistémologie. On sait que dès l'article "Sur le jeune Marx » (La Pensée, 1961), le projet global est en place (reformulation dans "Aujourd'hui ", 1965). Il n'a jamais varié.

- Primo : l'enjeu. C'est la mise à jour du marxisme français, resté scandaleusement romantique et arriéré par rapport aux recherches européennes. Pour la réussite du projet révolutionnaire, il est nécessaire de dépasser le marxisme vulgaire ou doxologique par la théorie. 
- Secundo : la méthode. C'est le retour au texte de Marx pour en faire une lecture symptômale permettant d'en rendre explicite le contenu latent, encore inaperçu. Althusser choisit la ligne scientifique contre la ligne éthique, affirmant qu'il suit en cela le cheminement du texte lui-même. À première vue, il apparait que les deux registres s'entrecroisent dans le corpus marxien; déjà, la fin du dix-neuvième siècle a vu des affrontements entre les deux interprétations, en Autriche, en Allemagne et en Russie. Pour sa part, Althusser donne priorité à l'éclaircissement d'une ambiguïté qui a par ailleurs servi l'expansion pratique de la doctrine de Marx. Il procède d'abord à une répartition chronologique des œuvres selon la séquence idéologie/science, situant la coupure en 1845, comme chacun sait. Il opère ensuite une hiérarchisation, posant la science marxienne comme discours de la maturité, achevé, autonome et surtout novateur. C'est d'une science normative qu'il s'agit. Par cette double intervention, Althusser borne le marxisme scientifique à un nombre limité de sources et lui assure une cohérence effectivement plus stricte.

- Tertio : le programme. L'entreprise s'inscrit d'emblée dans une visée programmatique cumulative dont les étapes sont ainsi définies : d'abord circonscrire le concept de science par un retour/recours à Marx et quelques emprunts à la psychanalyse ; élaborer ensuite la science de l'idéologie pour démasquer l'illusion coupable et dissoudre avec elle les faux problèmes qui encombrent l'horizon du champ théorique ; enfin, se tourner vers l'objet privilégié de la pratique scientifique : l'histoire, ce « continent histoire » encore inexploré.

À ce jour, la démarche d'Althusser n'a pas atteint le terme de la seconde étape. Or, les deux premières ont le statut de prolégomènes à toute science future. La science marxiste comme telle reste donc à construire. Rien ne donne à penser que cette immense tâche sera effectivement réalisée dans les années à venir. S'agit-il d'une impossibilité théorique ou d'un passage au purgatoire?

S'il est évident qu'Althusser n'est pas Hegel et que sa Grande Logique n'a pas encore été écrite, il reste que nous avons déjà une sorte d'homologue de la Phénoménologie de l'esprit. Le parallèle n'est pas gratuit : dans les deux cas, c'est l'anthropo- 
logie du sujet intentionnel que l'on fait éclater, chez Hegel pour la dépasser, chez Althusser pour l'écarter. Dans ce travail de sape, l'écriture fragmentée d'Althusser a apporté une contribution considérable aux recherches de l'après-guerre. Que l'on soit ou non marxiste, il faut compter avec le progrès réalisé dans la connaissance de la thématique marxienne grâce à la tribune de la rue d'Ulm. Il est pourtant impossible d'accepter l'affirmation d'« Aujourd'hui » (préface à Pour Marx, 1967) selon laquelle les intellectuels français auraient paresseusement négligé les textes marxiens, se contentant d'enseigner un Marx hégélien ou un Marx husserlien. C'est un signe que le Paris des années soixante a continué d'ignorer la province et s'est ainsi privé de ressources fort riches. On pense, entre autres, aux travaux remarquables et suivis du Centre de Recherche et de Documentation sur Hegel et Marx, rattaché à l'Université de Poitiers et dirigé par Jacques D'Hondt.

Cette réserve faite, il y a l'intérêt et l'originalité des articles historiques et du décodage du Capital (dont la facture collective pratique une grille unique). C'est sur cette matière que la catégorie de problématique, empruntée à Martin, montre le mieux son efficace opératoire. En effet, Althusser scrute un certain dix-neuvième siècle en y établissant des réseaux clairs, lesquels sont eux-mêmes rapportés à leurs sources respectives dans la pensée des Lumières. Sa recherche suit ici les seules trajectoires de sens qui entrent dans l'instauration de la modernité. La répartition des attaches marxiennes renouvelle les questions autant que les réponses : le lecteur est entraîné dans une expérience dont l'audace rappelle la rencontre de Heidegger avec Kant, la naïveté détachée en moins. Mais ici, ce sont surtout les arêtes du discours « relu » qui sont jugées porteuses de signification et amplifiées avec vigueur. La périodisation des écrits et manuscrits marxiens peut être contestée et elle l'a été ; cependant elle garde sa qualité historique éminemment érudite et rigoureuse. Peutêtre le « cloisonnisme " pesant de cette systématisation masque-t-il quelque peu la fluidité de l'écriture de Marx. Car, est-il vraiment pertinent de vouloir prouver que l'inachèvement du Capital répond à une nécessité épistémologique interne? Il reste que l'ensemble des résultats ouvre une voie et 
qu'on ne pourra plus employer dorénavant la notion d'aliénation à toutes les sauces, par exemple.

D'autre part, Althusser a cherché à se donner des instruments plus fins que ses prédécesseurs. Les plus intéressants, parce que les mieux maîtrisés et les plus féconds, pourraient bien être les idées de formation sociale, de problématique, de champ et de dominance, ainsi que la décision de réserver le terme de «notion» pour désigner les contenus idéologiques, celui de "catégorie" pour ceux de la philosophie et celui de " concept " pour les contenus scientifiques. L'auteur y gagne beaucoup en lisibilité. Quant à déterminer ce qui, dans cette écriture inégale, ressortit à l'emprunt ponctuel, à l'influence osmotique plus ou moins contrôlée ou à l'appropriation active, ce serait l'objet d'une autre étude. Pour des raisons qu'on exposera plus loin, le lexique structuraliste soulève des problèmes de cohérence dont certains avaient déjà été reconnus par Sebag, dès 1964 (dans le cadre non spécifique de la tradition marxiste, il est vrai). Ces problèmes, Althusser ne les a pas résolus. Ils restent donc à l'ordre du jour. Le recours au lexique psychanalytique est encore plus délicat puisqu'Althusser emprunte non à Freud mais à son collègue Lacan: ce détour s'apparente donc partiellement à la difficulté précédente. Le jeu des « transferts" lacaniens a été circonscrit avec précision dans une étude de Dufour (Culture et langage, 1973). Sauf pour quelques incidences sur l'idéologie, on ne s'engagera pas dans cette direction au cours des présentes réflexions car cette question déborde largement le terrain épistémologique auquel on entend limiter cette enquête. Et comme notre objet est la thèse centrale de l'opposition science/idéologie, voyons sur quelle topologie de la conscience celle-ci se pose.

\section{LES FONDEMENTS DE L'OPPOSITION SCIENCE/IDÉOLOGIE}

Althusser est marxiste. Or sa thématique est tout entière inscrite dans le champ symbolique, c'est-à-dire dans l'ordre du reflet, de l'effet, du dérivé, du déterminé. Le cadre général du matérialisme est certes verbalement posé et le déterminant économique constamment évoqué/invoqué. Mais il n'est nulle 
part analysé. Les rapports de cause à effet ne peuvent pas être contrôlés, vu le choix de l'objet. Nous aurons donc une science sans lois. Dans ce discours, le modèle ne se déploie jamais dans l'ordre réel qu'il postule, soit du déterminant matériel au déterminé idéel. Pour emprunter les catégories d'Aristote, les articulations du texte suivent les contours de l'ordre épistémologique sans qu'un ordre ontologique y soit terme à terme opposé. Le premier membre de l'équation est volontairement isolé par une sorte d'épochè indéfiniment prolongée. C'est que l'auteur se ménage explicitement un lieu transcendantal d'où il pense les conditions de possibilité d'un appareil conceptuel invulnérable à la sournoise déraison. Cette position du discours, suspendu au-dessus de la mêlée, lui a valu les accusations de kantisme et de théoricisme, venant de marxistes traditionnels tels Rancière, Vincent ou Mandel.

Il y a effectivement deux problèmes ici. D'abord, en dehors des trois instances économique, sociale et idéologique, quel est le statut de cette "pratique différentielle à caractère théorique" qu'est la science? Et en quel sens peut-on aujourd'hui parler de $L A$ science ? Cela rappelle le problème des hypostases chez Plotin où il faudrait décider si la matière constitue objectivement une quatrième hypostase innommable ... On voit où les objections du marxisme doxologique veulent en venir: il n'y a pas de lieu neutre; les motifs méthodologiques ne font qu'aggraver ce cas d'illusion idéaliste ; c'est une forme vulgaire de l'idéologie universitaire. Second problème : tant que l'instance économique n'a pas été développée par des enquêtes, quantifications et vérifications expérimentales capables de circonscrire avec exactitude la situation objective contemporaine, quelles garanties de validité offre cette science à laquelle manque le facteur décisif de rectification? En attendant, il est impossible de préciser ce que la robotisation des usines de montage japonaises ou la réévaluation des réserves pétrolières algériennes changent dans les idéologies particulières. Quels seraient exactement les vices réactionnaires qui provoqueraient les égarements des ouvriers polonais, dans leur lutte contre le parti communiste? Autrement dit, il n'est pas évident qu'on puisse formuler une "vérité concrète " en faisant la science des superstructures 
avant celle des infrastructures. De par les objets qu'elle choisit, l'écriture d'Althusser produit un colosse aux pieds d'argile. L'entreprise de Jacques Attali est indéniablement plus fidèle à Marx : en s'attaquant à la crise économique, ce dernier revient à une objectivité mesurable et opposable aux représentations.

Après avoir souligné quelques-unes des difficultés fondationnelles liées au lieu d'où parle cette voix, nous changeons d'échelle pour examiner les fondements plus immédiats de la corrélation entre idéologie et science. Ces fondements, il faut les chercher au niveau de deux autres couples de notions. On trouve d'abord les corrélatifs imaginaire/réel qui véhiculent une surprenante psychologie des facultés, de type classique ; en conséquence, la conception des corrélatifs erreur/vérité chez Althusser demeure tout aussi étrangère à la pensée dialectique. Commençons par le premier couplage. La position et le rôle de l'imaginaire commandent le système. Quoiqu'aucun chapitre n'y soit consacré, l'imaginaire est partout apparenté au même manque : illusion, aveuglement, résistance, irréalité, bref dérèglement des fonctions cognitives débouchant sur le néant. Althusser assume que c'est une faculté tournée vers le corps et sẹs intérêts (Pascal) et qu'elle se déchaîne quand la volonté outrepasse la finitude de l'entendement (Descartes). Il nous le confirme en faisant de la raison la seule faculté qui ait prise sur le réel, tandis que la «folle du logis » menace sans cesse de faire irruption dans le délire passionnel. À ce compte, la conscience mythique des cultures archaïques ne serait même pas pré-conscience mais seulement imposture et chaos, muthos résistant indûment aux justes prétentions du logos. (Que diable Labrousse allait-il faire dans cette galère ?). Ce n'est pas forcer le texte d'Althusser que d'y repérer une telle polarisation. Bien entendu, les intérêts douteux qui sous-tendent et alimentent l'imaginaire sont ici des intérêts de classe. Toutefois, l'usurpateur a bel et bien reçu le sceau cartésien. Ici, l'imaginaire est " conçu comme un système de représentations qui sépare un sujet de la vérité " (c'est Rancière qui souligne en 1969, dans "Pour mémoire ») Comment ne pas regretter qu'Althusser ait aussi peu retenu de ses lectures bachelardiennes? Le concept de coupure épistémologique se trouve fort dépaysé dans cette psychologie tributaire du dix-septième siècle. 
Or, la seconde corrélation amplifie la première et confirme le rationalisme primaire qu'elle faisait entrevoir. Quelle est, en effet, la conception de l'opposition erreur/vérité dans la pensée classique ? On y postule, comme chacun sait, que la vérité est accessible. La conscience est capable d'une appréhension pure de l'être. Il y a une seule conscience vraie, universelle et normative, mais diverses consciences fausses, dont la fausseté est déraison. Inutile de s'attarder à l'inconsistance de l'opinion ou du préjugé, sur lesquels le doute exerce une prise dissolvante : le savoir cartésien, sitôt élaboré, les supplante sans en laisser de trace théorique. L'erreur, l'apparaître, la surface, le relatif, le singulier n'ont pas même la densité nécessaire au maintien d'une tension de paradoxe. Car la catégorie de l'erreur, qui les subsume tous, désigne un mirage ou un rêve (au sens pré-freudien, évidemment.). Pure fiction, l'erreur est définie, ou plutôt contre--définie comme l'effet transitoire d'un hasard dont il n'y a rien à dire : il n'y a pas de science des ombres. Il est clair qu'au début de la modernité, l'erreur appartient encore au registre du non-être. Et si on le rappelle ici, c'est qu'en dépit d'un lexique nouveau, le discours d'Althusser opère, dans la plupart des cas, selon ces vieilles catégories ontologiques qu'il reproche à ses adversaires d'utiliser. Sans autre forme de procès, l'opinion est devenue la culture, tandis que le préjugé s'appelle l'idéologie, comme le remarque Dumont (Les idéologies, 1974). Parce qu'elle éclaire les rapports entre science et idéologie, nous retenons cette quête nostalgique d'un autre Discours de la métbode, réalisant l'idéal de la transparence rationnelle dans l'expression d'une vérité - adéquation. C'est là le paradigme qui régit l'idée de la science althussérienne. Toutefois, le discours in'est pas homogène sur ce point ; nous traiterons plus loin les motifs mineurs qui s'en démarquent et l'impossible « articulation » du tout.

Autre problème fondationnel : le rôle de la thèse matérialiste, car cette vérité - adéquation doit encore dire à quoi elle s'égale. Contrairement à la problématique de Marx, celle d'Althusser n'a pas besoin d'un postulat matérialiste pour se déployer. Elle y a pourtant fréquemment recours, mais c'est d'une manière qu'on ne peut qualifier autrement que d'opportuniste. En fait, l'affirmation du primat de l'être sur la pensée 
n'intervient que sous deux formes, également gratuites et autoritaires : d'abord comme argument visant à disqualifier la conscience (et singulièrement, celle du lecteur sceptique); ensuite, comme double fond dans un réductivisme facile: l'instance économique sert de crypte où sont reléguées la question des origines aussi bien que toutes les données dont la grille d'Althusser ne peut rendre compte.

Considérons le premier cas. En prenant appui sur un matérialisme abstrait, Althusser polarise le champ symbolique vécu autour de la notion de conscience fausse. Le décret primus positionne le poids ontologique de telle façon que le décret secundus puisse frapper tout le reste d'irréalité naïve. Le matérialisme élimine le hasard de cette irréalité : l'erreur est déterminée, prévisible, déductible d'un état matériel du système qui en est comme le fond nouménal. Mais ce discours est-il vraiment aussi kantien qu'on l'a agressivement relevé ? Nous pensons qu'à certains égards, le recours au matérialisme le couperait plutôt de la fécondité de la révolution copernicienne. Chez Kant, en effet, la décentration de la connaissance n'est pas le symétrique d'un processus de déréalisation. C'est l'inverse : l'écart entre l'ordre nouménal et celui du phénomène permet au travail constructif de s'exercer : cet écart s'interprète comme la condition de possibilité du discours vrai. L'autonomie normative de la subjectivité kantienne fonde la libération des formes, désormais détachées de tout asservissement référentiel univoque à la métaphysique. C'est en donnant la formule de ce changement d'axes que Kant lève l'obstacle majeur à l'émergence de l'épistémologie comme discipline distincte de la psychologie de la connaissance. Pour Althusser, au contraire, l'écart doit être annulé (si l'on excepte provisoirement quelques paragraphes qui tiennent un autre langage). Il ne devrait pas y avoir de distance, et s'il y en a une, c'est qu'elle est produite par une distorsion de l'instance économique. Cet intervalle surdéterminé entre la conscience et le réel c'est précisément le champ idéologique, dans le modèle althussérien. C'est dire que la conscience phénomènale représente, par un jeu fantasmatique de présence/absence, la contrefaçon d'un noumène sur lequel la théorie juste aura la décence de se régler. On le voit, ce pseudo-kantisme n'est pas 
assez agnostique : il n'admet pas l'obscurité d'une raison qui échapperait à la prise théorique de l'entendement : on voit que le rôle du postulat matérialiste consiste à poser l'invincibilité de la pratique scientifique, à ce premier niveau.

Le paradoxe ne s'arrête pas ici ; ses conséquences compromettent la cohérence globale du modèle. En effet, dans l'entreprise de dissolution radicale de toute subjectivité, les modalités du discours althussérien se trouvent à exclure les contenus que la dialectique est censée dépasser en les conservant. La démarche perd alors son moteur, en quelque sorte. Historiquement, il y a une première déperdition du multiple entre Hegel et Marx. Ce que Hegel entend par "culture " (dont l'homologue althussérien serait la "problématique") c'est la séquence des phases d'aliénation qui diffracte les progrès temporels du savoir en une multiplicité de facettes symboliques. Dans l'optique qui nous intéresse ici, la Pbénoménologie détaille la positivité résistante et riche des formes, disséminées par l'historicité. Ces œuvres de l'esprit ne tombent pas sous le critère cartésien du vrai/faux. Il n'y a pas d'intérêt, selon Hegel, à les désavouer. Tout rationaliste qu'il soit, Hegel reconnaît prima facie la vérité de la religion, de l'art et de la philosophie. Vérité d'expression, où se lisent les créations et conquêtes successives du sens assigné à des mondes spécifiques. Il est clair que ces objets requièrent plus qu'une simple curiosité historique : ce sont les matériaux de la science hégélienne. Nonobstant le point de vue du savoir absolu, Hegel relit ce qu'Antigone répond à Créon pour ce qu'elle dit, autant que pour ce qu'elle ne dit pas. Or, chez Marx, la vis est serrée de plusieurs tours : l'effet se résorbe plus nettement dans la cause. Puisque la totalisation se fait à partir de la catégorie de travail, la production est élevée au statut d'événement réel. L'autre du réel, c'est le reflet. Il a encore pour Marx la consistance minimale de l'image dynamique. Althusser ferme le cercle. Sa lecture symptômale ne retient de Marx (et a fortiori de toute ouvre) que le non-dit, c'est-à-dire la science à faire en revenant au degré zéro de la subjectivité. Marxisme binaire plutôt que dialectique qui ne cherche pas le dépassement mais la régression vers une zone originaire sûre (Auzias, Clefs pour le structuralisme, 1967), pré-œdipienne, pré- 
idéologique. Faut-il comprendre: préhistorique? Pour Althusser, tout se jouerait effectivement ailleurs. Car d'emblée, les superstructures de notre formation sociale sont déclarées malades de la peste. De proche en proche, la contamination a compromis tout le champ de la représentation. L'idéologie est non seulement politique, sociale et religieuse, elle est aussi militaire, juridique, pédagogique, économique, épistémologique, sexiste, socio-biologique, écologique, etc. sans parler des variétés internes au socialisme telles le gauchisme, le culte de la personnalité, l'humanisme socialiste. "Ils ne mouraient pas tous mais tous étaient frappés ". Ainsi tous les rapports de la conscience à ses objets seraient irrémédiablement pervers en même temps que dérisoires. Seule la science est capable de les débouter et de les remplacer par la pensée correcte. Seule elle peut départager la bonne conscience de la mauvaise. Finies, les médiations hégéliennes : la mutation s'opère par l'illumination d'une conversion postulatoire, accompagnée d'une amende honorable (et du ferme propos, sans doute). Étrange marxisme qui, d'un côté, en appelle au matérialisme pour démasquer les bévues, et de l'autre, s'en passe fort bien pour poser une science qui transcende l'histoire et la révolution. Le privilège théorique semble tenir de la " window-pane theory "implicite à toute rhétorique savante. C'est ce qu'ont récusé plusieurs critiques qui avaient d'abord accepté le cadre de ces recherches; se sentant acculés au choix, ils sont retournés au marxisme orthodoxe d'où ils étaient venus. Cela ne va pas sans rechutes comme le démontre l'ouvrage de Laurin-Frenette, Production de l'État et formes de la nation (1978), dont les deux premiers chapitres dénoncent Althusser cependant que la suite reproduit la thétorique de la reproduction.

L'autre difficulté que pose le recours à la thèse matérialiste dans l'écriture d'Althusser pourrait se nommer " généticisme ", par où il ne fait qu'accentuer un problème du marxisme traditionnel : celui de la genèse des superstructures. Au fondement de l'opposition science/idéologie, il y a une opposition dans leur mode respectif de production. La science marxiste, en effet, échappe à la contamination par le privilège de la raison : elle est théorie de la problématique propre à une formation sociale et, de ce fait, hors du champ idéologique. 
Tout le reste est superstructure, assignable à l'instance idéologique, elle même produite selon les lois du matérialisme dialectique et donc déterminée par la lutte des classes. Althusser pratique, comme on le voit, un matérialisme sélectif. Mais là où celui-ci s'applique, il s'applique radicalement et sans pitié. Car toute erreur est suspecte : elle est d'abord mystification et alibi (au sens moral aussi bien que locatif). Sur le plan épistémologique, le modèle althussérien est hyper-réducteur, en dépit de la catégorie d'autonomie relative. Le seul champ autonome, en fait, est le no man's land de la théorie où prévaut l'ordre des compétences ("Problèmes étudiants", 1964). Et comment opère cette théorie? Elle simplifie les diverses strates du vécu (singulier comme collectif) et escamote les lignes de l'irrationnel dans un modèle hypothético-déductif universaliste. Tout s'explique, en dernière instance, par la lutte des classes sur laquelle, pourtant, aucune analyse descriptive spécifique n’a été produite : il faut s'en remettre à Marx et à Lénine sur ce point capital et il se trouve justement qu'ils n'ont rien laissé de scientifique ni définitif sur le sujet (Aron, La lutte de classes, 1964). Qu'à cela ne tienne! La science d'Althusser est tellement abstraite qu'elle réduit à la fois l'histoire, les sociétés, les représentations ainsi que les objections qu'on pourrait oser lui faire. Sur ce dernier point, les adversaires de la vraie foi sont rapidement classés par le Saint-Office : païens, hérétiques ou relaps. On en voit une démonstration dans ce que Rancière appelle trop poliment la «leçon d'orthodoxie " infligée à John Lewis et dont le ridicule est certainement le trait le plus saillant. Par exemple, si l'on objecte que la matérialité comme telle est absente de ce matérialisme, puisque l'autre de l'idéologie, ce n'est ni la matière, ni le rapport concret à la matière, mais bien une science économique, seconde, codifiée, homogène au champ de la représentation, ou, pire encore, une instance inconsciente, diffuse et fuyante (ce qui dispenserait le théoricien marxiste de compétence économique : il n'aurait plus qu'à pratiquer la psychanalyse...), on connaît d'avance la réponse: l'adversaire n'a pas bien compris le matérialisme correct. "La théorie donne sa propre répétition pour l'analyse de son objet. " (Rancière). 
La maîtrise des modèles réducteurs n'a pas non plus fait de progrès scientifiques dans les textes d'Althusser ou de Badiou. C'est pourtant une question épistémologique intéressante en soi et certainement prioritaire pour assurer les fondements du marxisme. Il ne va pas de soi qu'un système de représentations symboliques est l'effet ou le produit d'une organisation empirique spécifique. Comment est conçue l'intersection entre les deux registres? Faut-il s'en remettre à d'inconscients esprits-animaux dont les pouvoirs de détermination tiennent entièrement à la vertu d'un hypothétique trait d'union? Mais ce n'est pas seulement dans la pratique réductiviste comme telle que la faiblesse théorique se manifeste. C'est dans l'oubli du fait fondamental suivant: la réduction est une opération intellectuelle de subordination logique et non d'abolition. Cette hiérarchisation ne supprime pas les distinctions et chevauchements entre les divers ordres d'expérience dont aucun déterminisme ne saurait rendre compte. En effet, le réel n'est pas "pliant": ce sont les concepts qui constituent un modèle télescopique. Une fois le concept cartésien de paraffine élaboré, le chimiste, même aveugle, peut certes en parler scientifiquement ; le morceau de cire est néanmoins toujours sur la table, visible, et cela avant, pendant, comme après la démarche scientifique. Sa présence sensible n'est pas fictive, ni la perception spontanée que j'en ai, ni l'usage empirique que j'en fais. Un discours incarné exprimant les rapports de la conscience aux gens et aux choses n'est pas vide de sens ni pure mystification, comme en témoignent de récents essais sur le discours amoureux ou le regard du photographe (Barthes). Le réductivisme confortable et omnivore d'Althusser a choisi la disqualification radicale du vécu de la conscience. En conséquence, il se croit dispensé de considérer la simple collection des phénomènes de représentation. Il n'y a pas de place, dans le discours d'Althusser, pour une morphologie du champ symbolique ni pour les syntaxes régionales des idéologies et des cultures (Que diable Labrousse allait-il faire dans cette galère ?). On voudrait ici s'inscrire en faux contre cet immanentisme tranquille qui digère toute apparence. Il occulte le singulier, ses tensions et son ouverture permanente sur le désordre. L'instauration de ces deux ordres que sont la science (raison théorique) et l'idéologie (raison 
pratique) n'a de sens que comme entreprise pour échapper à ce vertige et le tenir à distance. C'est pourquoi la pensée est autre chose qu'un luxe d'universitaire. Avec tout son moralisme infantile, Sartre oppose du moins l'existence à la structure; pour Althusser, c'est le réel lui-même qui fournit immédiatement la structure et la théorie (Auzias, 67), qui est structure et théorie. Drôle de matérialisme, en vérité, et drôle de science qui n'a plus d'objet à élucider, s'étant enfermée en elle-même et niant le dehors comme on le faisait au jardin d'Épicure. À nos yeux, pour satisfaire aux exigences de la scientificité, il faudrait que soit a priori posé l'être de l'apparaître, c'est-à-dire la réalité, la vitalité et l'expressivité dynamique de la représentation "sauvage ", à laquelle une relative autonomie ne suffit pas. Pour qu'il y ait discours scientifique, l'objet défini doit être cognitivement discernable et séparable des autres objets. Il faut identifier un effet autrement que comme un redoublement de sa cause. Althusser, épistémologue, n'a pas plus qu'un autre le droit de départager le vrai réel du faux réel; la réduction totalitaire n'est pas un argument scientifique.

Nous avons examiné trois problèmes fondationnels : le statut de la science althussérienne, les notions corrélatives sous-jacentes à l'opposition science/idéologie et le recours au matérialisme. Les insuffisances théoriques relevées compromettent la cohérence de la thèse centrale dont nous verrons maintenant les termes.

\section{LA SCIENCE COMME STRATÉGIE CONTRE L'OFFENSIVE IDÉOLOGIQUE}

Althusser, on le sait, a peu écrit. Même en comptant les polycopiés de Normale, la somme des pages n'approche pas le quart de l'œuvre de Bachelard ou de celle de Lacan. De plus, il n'a pas encore publié de somme développée où les articulations se montreraient ensemble, en ce qui concerne la première phase de son programme : l'élaboration du concept de théorie. Cela ne facilite pas l'étude de la seconde phase, la théorie de l'idéologie, qu'il faut chercher dans divers articles, préfaces et notes du traducteur. Elle est considérée comme la contribution majeure de l'auteur et c'est à elle qu'il doit sa célébrité. Nous 
l'étudierons ici à partir de ses prétentions à la scientificité, en commençant par quelques remarques sur le contexte d'où cette théorie de l'idéologie a émergé et sur ce qu'elle doit aux sciences humaines qui lui servent d'horizon. Nous verrons quelles sont les règles qui gouvernent la construction de l'objet-idéologie dans ce discours.

En premier lieu, le contexte. On savait que, depuis Feuerbach, il n'y avait plus moyen de "faire " de l'anthropologie sans compter avec le discrédit de la conscience. On sait maintenant que le point de vue anthropologique lui-même a été liquidé, même dans la grille marxiste. Au début des années soixante, la gauche décrète que tout est politique. On découvre que Nietzsche, Freud et Reich sont d'abord des penseurs politiques. La politisation objective de tous les secteurs de la vie est disséquée par Marcuse, Deleuze, Morin, Habermas, Kuhn, Chomsky. Ce mal qui répand la terreur affecterait donc toute représentation et tout discours ; cette grande découverte aurait une portée historique rétroactive. On doit pourtant élever une objection élémentaire ici, dans la mesure où il y a une histoire de la place que la culture occidentale a assignée au politique. Fort étroite dans la représentation du Grec, elle s'est élargie à la Renaissance en corrélation avec la montée du phénomène urbain (Ellul). C'est la modernité qui a ouvert les vannes à l'envahissement du privé par le socio-politique en produisant une image profane et acosmique de l'homme (Arendt). Cette image amplifie dans le champ symbolique l'appartenance à un groupe abstrait : plus les liens concrets, personnels et familiaux, vont se dissoudre, plus l'investissement dans le politique va les récupérer et déséquilibrer la représentation. Ainsi, la sphère du politique, telle que nous l'entendons aujourd'hui, n'est pas une donnée "objective ", encore moins éternelle; c'est un indice culturel récent et géographiquement limité. De plus, on n'a jamais établi que cette extension globalisante de la représentation du politique coïncide avec un accroissement de l'effectivité mesurable de l'action. Au contraire, il apparaît que l'action effective, individuelle ou collective, doit être conçue comme un invariant historique, comparable à la part de l'irrationnel. Il n'y a pas lieu de développer ici cet aperçu. Je voudrais seulement 
montrer que le postulat "pan-politique » est loin d'aller de soi. Il n'explique rien et c'est plutôt lui qui appelle des explications. Or Althusser partage ce postulat commun aux discours marxistes : il compte comme évidence rationnelle ce qui est une évidence culturelle (plutôt que fausseté ou bévue). Quand on tient que tout est politique, il s'ensuit qu'on fait de la politicologie sans tenir compte des champs disciplinaires dont les frontières ne sont que des effets idéologiques consolidant le pouvoir des experts. Badiou ne nous dit-il pas qu'il faut démasquer l'épistémologie bourgeoise dominante? (Le concept de modèle, 1972). L'impérialisme théâtral du politicologue s'octroie ainsi la propriété privée de l'idéologie.

C'est sur le terrain de ces luttes qu'Althusser poursuit ses recherches. Cela éclaire les conditions épistémologiques de la construction de l'objet-idéologie, dans son discours. Le repérage précis du phénomène a été négligé au profit d'une symptomatique de la fraude. Cette position implique que rien ne se joue dans l'idéologie. Elle n'est pas un lieu de lutte donc c'est un non-lieu. Alibi, disions-nous : en effet, tout se joue ailleurs et l'idéologie sert de diversion, masquant les forces en présence par un fantasme dont elle ne possède pas la clef. Il faut la combattre par la science marxiste qui a ici deux rôles : premièrement rectifier la conscience de la lutte en la déplaçant vers son objet réel; deuxièmement donner la clef de la mystification/justification. En s'appuyant sur l'autre de l'idéologie qui est science des conflits, la raison rend compte de la rationalisation. On fait l'économie de la définition de l'objet en le traitant comme pure fonction de relais du pouvoir. La fonction pratico-sociale l'emporte sur l'aspect cognitif.

Il n'y a pas ici l'ombre de l'attention que Freud accorde à un rêve de son patient, en tant qu'élément porteur de sens intrinsèque, par exemple. La théorie de l'idéologie, sous sa forme achevée, affirme que "dans l'idéologie est donc représenté non pas le système des rapports réels qui gouvernent l'existence des individus, mais le rapport imaginaire de ces individus aux rapports réels sous lesquels ils vivent. "(Idéologie et appareils idéologiques d'État, 1970). Le fonctionnalisme est évident : la conscience idéologique est un effet des rapports de pouvoir. Or le pouvoir est maudit quand il est capitaliste et 
bourgeois. L'intention éthique se substitue ici à la visée scientifique. L'appareil théorique renonce à la typologie, à la sémantique et à la logique des formes idéologiques pour se borner à montrer les intrigues souterraines et inavouables de la classe dominante avec le pouvoir. On connaît bien les épisodes dramatiques des rapports ambigus qu'entretiennent, volens, nolens, les intellectuels français avec le pouvoir politique (ou, plus exactement, avec l'image qu'ils s'en font.). Comme l'idéologie qu'Althusser cherche à circonscrire est celle-là même que ces intellectuels véhiculent, il introduit, dans la définition, des coefficients de valeur défensifs qui expriment la tension interne et la méfiance. En conséquence, la subjectivité n'a pas été traitée à la troisième personne : elle n'a pas été constituée en objet. L'opposition science/idéologie se bloque dans la dichotomie sacré/profane. Il s'ensuit que l'idéologie a d'abord été dite bourgeoise et dominante, mesquine et répressive, avant même d'être quelque chose. On ne sait pas encore ce qu'elle est, sinon qu'il faut la penser "sous le concept d'exploitation" (Réponse à John Lewis, 1973).

Certes, la définition est décisoire: Althusser a le droit indiscutable de proposer la sienne. Et on tente ici d'en mesurer l'efficace opératoire. Le problème, c'est qu'il ne définit pas mais qu'il délimite : est idéologique ce qui n'est pas scientifique. "L'idéologie ici est pensée comme fourre-tout : on y met tout ce qui n'est ni science ni épistémologie » remarque Mikel Dufrenne (Pour l'bomme, 1968). À propos d'idéologie, Claude Savary nous rappelait avec humour, lors du récent colloque de Trois-Rivières, que, selon Perelman, la philosophie est l'étude systématique des notions confuses. La science althussérienne semble avoir trouvé un moyen de s'en débarrasser tout en prétendant occuper tout le champ de la philosophie. C'est peut-être ce que les clients demandaient mais ce n'est pas assez pour satisfaire aux exigences minimales du discours scientifique. Contre toute attente (si l'on se fie à son programme), Althusser a bifurqué ici vers le marxisme doxologique le plus traditionnel. C'est que, pour objectiver rigoureusement la conscience idéologique, il eût fallu renoncer au cadre du matérialisme dialectique. Cette difficulté est fondamentale et donne la limite intellectuelle du marxisme présent et futur, 
sans qu'il soit besoin d'être prophète. Revenons au modèle. L'idéalisme métaphysique des essences est radicalement incompatible avec la doctrine de Marx et d'Engels. Les concepts véritables sont nécessairement des concepts critiques : ils énoncent des rapports (c'est bien ce qu'Althusser dit de l'idéologie). La dialectique précise qu'il s'agit de rapports de contradiction. Le matérialisme limite les contradictions réelles aux rapports de forces antagonistes générés par la production industrielle, pour une formation sociale capitaliste avancée. L'idéologie est un instrument de l'exploitation exercée par la classe dominante. Le seul référent objectif du concept critique d'idéologie c'est la classe (ce que reprend Althusser). On le voit, la marge de liberté spéculative est mince. Pour rester marxiste, le discours d'Althusser doit pratiquer ce réductivisme fonctionnaliste. Cette série de contraintes a un double effet théorique : d'une part, ce discours ne peut rendre compte que du versant pragmatique (contenu social) du phémonène idéologique ; d'autre part, il doit affirmer que c'est le seul point de vue correct et qu'il est exhaustif.

Sur le plan épistémologique, donc, cette construction produit un objet à refouler, indéfinissable par des règles internes d'intelligibilité. Cet objet-idéologie est symptôme de manque, de défaut, de trou dans la quête de la vérité. On l'a construit pour en faire le procès et c'est de procès d'intention qu'il s'agit. Car la lutte des classes n'est pas innocente. Chez Althusser - et la tendance s'accentue d'un texte à l'autre l'idéologie, c'est un vice dont on accuse son interlocuteur. C'est encore le JE et le TU qui en décident; le ON impersonnel du discours scientifique n'a pas pu arbitrer ce combat, dont l'enjeu, tout compte fait, n'est pas un enjeu d'intelligibilité, mais bien de salut. Il s'agit essentiellement de sauver la vérité en sauvant la société. Rien ne vient élucider ces rapports douteux entre vérité et rédemption. Et chaque fois que de tels rapports ont été postulés dans la pensée occidentale (pythagorisme, méso-platonisme, scientisme, etc . . .) cela a coïncidé avec un temps faible sur le plan de la rigueur intellectuelle.

Je souligne que c'est à lui-même que j'oppose Althusser. Son projet explicite vise à sortir du marxisme vulgaire ou 
doxologique, simple catéchisme de parti, par une science authentiquement marxiste, formalisée dans une pratique théorique transparente, s'élaborant sur le terrain où les sciences de l'homme définissent, décrivent, analysent, classent, expliquent et vérifient objectivement. Premièrement, il n'est pas sûr qu'il existe de telles sciences naturelles de l'homme. Deuxièmement, Althusser n'a pratiqué aucune de ces opérations. Il n'a pas réussi à construire une définition formelle du phénomène idéologique qui s'exprimerait dans une série d'artefacts linguistiques autonomes, par laquelle on pourrait procéder à des opérations. En essayant de tenir ensemble les impératifs formels de la scientificité et les impératifs catégoriques du parti communiste français, le discours althussérien véhicule (et differe autant qu'il peut) la plus plate, la plus stérile, la moins dialectique des contradictions. Contradiction prévisible, au surplus. Il a fallu choisir: c'est le moralisme qui a prévalu. L'idéologie-relais de la lutte des classes prétend se déplacer vers le champ de la science. Dans son apologie naïve, Karsz nous en donne la formule : «Or, à relire Althusser aujourd'hui (1974), on s'aperçoit que l'appel à la Théorie fonctionnait politiquement. ». (Souligné dans le texte). D'un côté, l'idéologie dominante est mauvaise parce que bourgeoise ; elle renforce l'exploitation en imposant au dominé sa place dans l'appareil de production, par une contrainte indue. De l'autre côté, la science est bonne par son intention de libération du prolétariat. Encore Karsz: «Mettre l'accent sur l'aspect théorique de la politique, sur le besoin d'une théorie fondée pour une politique de classe juste. » (C'est encore le désarmant Karsz qui souligne.) C'est exactement ce qui fait problème, sur le plan épistémologique. Pour emprunter à Quine, nous dirons que la dimension formelle de l'idéologie est traitée par Althusser comme une fonction de l'empirie. Encore faut-il ajouter qu'il s'agit de l'empirie du sentiment moral de la justice. Nous sommes plus près de Sartre que les premisses althussériennes le donnaient à penser. Pour le spectateur, l'intérêt de l'entreprise réside dans la démonstration des limites indépassables du modèle marxisteléniniste dans sa version la plus « savante », c'est-à-dire la version théologique. 


\section{LA STRUCTURE : UN EMPRUNT INDIGESTE}

Nous avons ignoré jusqu'ici un registre distinct dans lequel la théorie de l'idéologie s'exprime secondairement quoique d'une façon récurrente : le ton structural. Il présente, sans arriver à la fonder, la distinction entre l'instance idéologique et les idéologies spécifiques. On en trouve la formulation explicite en trois lieux principaux. Il s'agit de «Marxisme et humanisme " (1964), «Théorie, pratique théorique et formation théorique » (1965), ainsi que «Idéologie et appareils idéologiques d'État» (1970). Sur la question de l'idéologie, le modèle dialectique examiné plus haut coexiste avec le modèle structural. Dans l'œuvre d'Althusser, ils ne se succèdent pas comme deux moments séparés par une coupure épistémologique. Cependant, le lecteur ne trouve pas la confrontation des positions ontologiques contradictoires (et d'ailleurs superflues, croyons-nous) qui y sont incompréhensiblement accentuées. Aucun des deux n'est renié ni relativisé en tant que logique régionale de l'autre.

Le recours à l'appareil structural constitue la grande nouveauté des travaux d'Althusser et leur a valu leur nom de marxisme épistémologique. Il a surtout servi à la lecture symptômale de Marx qui n'est pas visée ici. Comme chez Foucault, la pensée de la structure met l'anthropologie au ban du discours scientifique. Les termes de Quine s'inversent, par rapport au sens de l'argumentation matérialiste que nous venons de voir : dans la logique structurale, l'empirie est traitée comme une dimension du formel. La science s'isole et construit ses objets, sans avoir à fournir de lettres de créance, sans égard au vécu, en dehors et en deçà de l'idéologie. L'objet n'est pas à chercher ailleurs que dans sa définition. Le réel s'est donc déplacé sans s'en expliquer. Ce n'est peut-être pas là une question scientifique mais nous pensons qu'il faudrait en rendre raison au philosophe entêté. Car le décor épistémologique a changé. Tout n'est qu'ordre et discours: les infrastructures, la dialectique et la praxis ont disparu. Il n'y a plus de déterminisme, mais seulement " présence de la structure dans ses effets ", ce qu'Althusser appelle causalité métonymique ou surdétermination. La logique structurale est un système clos. On ne voit plus un seul trait qui distingue ce discours marxiste 
d'un idéalisme de la connaissance, se mouvant entre l'activité théorisante et ses concepts. Même la classique dénonciation de l'empirisme y est. On constate une sorte de transposition des motifs et moteurs concrets du matérialisme dialectique : la praxis, c'est la pratique théorique ; la fuyante nature, c'est le discours vrai ; la valeur, c'est la raison. Néanmoins, ce marxisme métaphorique ne reflète pas l'autre. La structure l'a dévitalisé en excluant toute considération de forces : lutte, résistance, conflit concret, domination, révolution, etc. ne peuvent être pensés par le langage structural. Ce dernier est construit pour les éviter et son efficace est ailleurs. Les problèmes de l'appropriation de l'emprunt sont rendus particulièrement épineux pour Althusser. Certains ont été signalés par Godelier, en 1966, (« Système, structure et contradiction dans Le Capital ») avec l'optimisme de celui qui croit que le travail commence. On voit aujourd'hui qu'il n'a pas été fait, et qu'il ne pouvait pas l'être. Pour ce qui concerne notre objet, la théorie de l'idéologie, l'incompatibilité est manifeste aux niveaux suivants.

1. À l'intérieur du modèle dialectique, Althusser a échoué dans la tâche qu'il s'était donnée: la formalisation de l'objet-idéologie. Ce qu'il demande à la structure, c'est la distanciation du regard scientifique permettant de dénouer les fils de la complicité sujet/objet. La structure l'accorde en abolissant le pôle sujet de l'objet-homme dont elle n'a pas besoin, sur le plan méthodologique. Le ON impersonnel structural n'est pas garanti autrement que par son succès dans un nombre limité de disciplines.

2. Il y a confusion sur le statut épistémologique de la structure elle-même. En effet, le raisonnement structural fournit des perspectives méthodologiques fécondes à la minéralogie, à la biologie et à la psychologie gestaltiste, par exemple. Dans ce cas, il s'agit de lectures d'une réalité donnée, extérieure au concept. La structure est devant le savant, comme système fini des transformations mesurables de ses éléments. Nous parlons d'une totalité observable, à découvrir et à reproduire formellement. Il n'en est pas de même en linguistique, ni en psychanalyse, ni en ethnologie. Ici, l'intuition structurale n'est rien d'autre que la règle d'intelligi- 
bilité entièrement liée au discours scientifique qui l'invente et en élabore des modèles logico-mathématiques. Cette structure est une catégorie herméneutique telle qu'on peut la simuler par ordinateur mais non l'observer. La structure althussérienne est de cet ordre.

3. À quel titre cet être imaginaire peut-il commander une vérité rationnelle puisque, selon le découpage althussérien des facultés, toutes les élaborations imaginaires portent des distorsions anthropologiques qui marquent un stade préscientifique? Le concept lui-même, où Derrida décèle la métaphore usée, n'est pas à l'abri de cette contamination. On voit l'incompatibilité des deux grilles. Encore une fois, où est la légitimité de cette science, comme absolu ? S'i l'imaginaire scientifique donne à penser (ce qu'il fait effectivement), pourquoi l'imaginaire idéologique ne le ferait-il pas aussi? Rien n'interdirait, pensons-nous, d'aménager des degrés d'apport cognitif. Mais Althusser s'en tient au système binaire du noir/blanc. Comme Dufrenne le remarque, l'œuvre d'art, le slogan, le sentiment religieux se retrouvent dans le même sac.

4. Le pas décisif, Althusser le. fait lorsqu'il durcit l'attitude méthodologique en ontologie. Cette fois, nous sommes dans le structuralisme. Le dernier mot sur l'être, c'est la pensée de la pensée, close sur elle-même dans une totalité théorique autosuffisante. Pour Althusser, la vérité de l'idéologie lui est immanente et contemporaine : c'est le concept latent qui attend d'être manifesté par la théorie. Nous avions, tout à l'heure, une première totalité, matérialiste et exclusive. En voici une seconde, idéaliste et tout aussi exclusive. Comment penser cet hiatus? Dans Le totémisme aujourd'bui (1965), Lévi-Strauss, qu'Althusser suit dans son type de structuralisme, exprimait son « refus du primat du social sur l'intellect ». Dans l'écriture oscillatoire d'Althusser, l'axiome de Durkheim reste indécidable. Et s'il devait y avoir une quelconque «articulation", ce serait au prix des deux postulats ontologiques auxquels se substituerait la pluralité des rationalités issue du pluralisme méthodologique : éclectisme bourgeois, qu'Althusser n'oserait suggérer. On reste donc dans l'impasse. 
5. Où est exactement l'efficace propre à la méthode structurale (qu'elle soit on non promue au rang d'ontologie) ? C'est de restreindre le point de vue à la considération de connexions stables pour faire apparaître le principe d'ordre en abolissant l'événement. "Les systèmes sont construits contre ce qui arrive. " note Domenach à propos des structuralismes (Esprit 350, mai 1967). En tant qu'il surgit une seule fois, dans une situation unique, un événement ne peut être objet de science parce qu'il n'y a pas de science de l'individuel. Ce sont les relations abstraites, atemporelles, répétables qui constituent le fait scientifique en permettant de le relier aux autres faits. Or le programme althussérien se définit par sa visée ultime: donner la première théorie scientifique de l'histoire, ce que Marx a indiqué sans pouvoir s'en donner les moyens. Il se trouve que la méthode structurale est aveugle au registre historique. Le choix d'Althusser est ici peu pertinent. Il y a erreur sur l'instrument ou sur l'objet. La science harmonique ne donne pas la mélodie, pas plus que la science contrapuntique ne donne les lois harmoniques.

6. Il reste à examiner la distinction althussérienne entre les idéologies historiques et l'instance idéologique. Nous avons vu que le matérialisme dialectique parvient mal à rendre compte de l'émergence et de l'inertie des idéologies particulières, la même cause déterminant des effets symboliques différents. Il ne fournit pas l'équation déductive de l'interface matériel/ symbolique. Le recours à la structure s'installe dans cet écart, à la suite de Lévi-Strauss. Le concept d'instance idéologique, tributaire du postulat structural dissout les liens de causalité. Ce terme d'instance a, lui aussi, une longue carrière puisqu'il apparaît chez Freud, dans L'interprétation du rêve (1900), comme synonyme de «système », avec une nuance de rigidité et d'autonomie plus nettes. Althusser pour sa part, l'emprunte, on l'a dit, à l'emprunteur Lacan qui l'a largement délesté de son énergie pour en faire une trame linguistique neutre. Le discours althussérien se montre à la fois laconique et répétitif sur le sens de cette entité : l'instance idéologique est identifiée au champ de la subjectivité. Elle est éternelle, coextensive au vécu des hommes et à la vie historique de toute formation sociale, même communiste. Ce n'est donc pas la 
lutte des classes qui l'engendre (si l'on suit naïvement Althusser dans la fiction de la disparition des classes en régime socialiste). Certains commentateurs ont cru que, par ce biais, il réhabilitait en pratique l'idéologie puisqu'il en faisait une dimension générale essentielle à la vie collective. Il n'en est rien. On ne trouve pas un seul énoncé qui préciserait en quoi l'instance idéologique enrichit les individus ou crée un sens. Sa permanence est plutôt une fatalité, un malaise dans la civilisation qui corrompt définitivement le paramètre homme par la faute originelle de l'irrationnel. Nul ne peut ni ne pourra sortir de cette caverne sans passer par le baptême de la scientificité. Le plus étonnant, c'est l'absence de lien avec la révolution. Que peut signifier cette instance anhistorique dans le marxisme le plus élastique ? Dans son modèle matérialiste, Althusser nous impose de penser l'objet-idéologie sous le concept d'exploitation. Il est clair que l'objet-instance, lui, ne peut s'y prêter. Le modèle structural introduit-il un nouvel objet ? Ou s'agit-il du même auquel on s'adresse autrement? Autre dilemne indécidable. Jusqu'à nouvel ordre, le discours althussérien pose l'instance idéologique au-dessus des idéologies particulières. Cette entité n'a pas de contraire; elle n'est pas issue d'une contradiction; elle n'est pas solidaire du dépérissement de l'État. Elle plane en un lieu suspect : celui que Marx assigne aux représentations idéologiques. Pour la fonder autrement, il faut sortir du marxisme (ce qui, après tout, ne serait pas un sacrilège. . .). Mais Althusser ne la fonde pas. Il s'affirme marxiste. Nous devrions nous satisfaire de quelques énoncés globaux sur les changements de niveaux et d'échelles. La production réelle est bien dans l'usine mais elle est aussi dans la théorie de la structure, laquelle n'a pas à se régler sur la première. Nous en concluons que les deux modèles ne sont pas articulables. De l'énergétique à la topique il y a solution de continuité.

Le lecteur jugera dans quelle mesure l'intention d'interroger la portée philosophique des thèses althussériennes se trouve remplie par nos questions. Jusqu'à ce point, notre démarche se voulait une critique interne du marxisme épistémologique comme contribution à la théorie de l'idéologie. Nous pensons avoir montré que, du réductivisme au scientisme, le problème 
de la construction de l'objet se complique et qu'il y a lieu de le juger insurmontable tant qu'il sera posé dans un tel cadre hétérogène non maîtrisé. La théorie althussérienne juxtapose des éclaircissements semblables à ceux des fameux épicycles du De revolutionibus: la représentation copernicienne impliquait plus de règles, d'exceptions, d'aberrations et de dispersion que celle de Ptolémée qu'elle cherchait à unifier.

Le bilan que nos propos autorisent met en évidence la nostalgie de l'impossible totalité. Alors qu'Althusser cherche encore la science des sciences, Bachelard avait vu que l'épistémologie fragmente et disperse nécessairement. L'histoire lui a donné raison: les pratiques épistémologiques deviennent progressivement internes à chacune des sciences, comme le constate Piaget (Logique et connaissance scientifique, 1967). Les artistes eux-mêmes laissent de moins en moins à d'autres le soin de dire comment penser leur esthétique. Les sciences dites humaines constituent le cas extrême. Si elles se montrent réflexives, écrit Dumont, ce n'est pas signe d'immaturité, c'est plutôt que le souci épistémologique est partie intégrante de leur visée scientifique. Par là, elles inaugurent une rationalité distincte de celle des sciences exactes. (Le lieu de l'homme, 1968). L'entreprise d'Althusser le confirme par l'absurde et la démonstration n'était pas inutile.

\section{QUELQUES AVENUES POUR PENSER CE QUE L'ÉCRITURE ALTHUSSÉRIENNE OCCULTE}

Sortons ici de l'assujettissement à tout impératif catégorique. En abordant le phénomène idéologique comme nœud des rapports concevables entre savoir et pouvoir, nous admettons, entre autres, la possibilité qu'il n'y ait pas d'articulation transparente entre eux. Il nous semble qu'en explorant le "versant positif » de la dimension idéologique, selon l'expression de Fernand Dumont, nous pourrions entrevoir une théorie plus équilibrée de la fonction symbolique, sans pour cela réhabiliter le préjugé. Plaçons-nous sur le terrain de la positivité, au sens où elle fait fond sur les données de l'expérience. À ce niveau, il faut renoncer à commencer par une théorie "critique " globale et s'attacher plutôt à élaborer un objet neutre, quitte à pratiquer une reprise critique ultérieure 
pour équilibrer les rapports de sens et les rapports de forces à l'œuvre dans le champ idéologique. Ainsi, la tentation de l'empirisme peut être en partie contrôlée pendant que la charge de culpabilisation est mise entre parenthèses.

Il n'est pas inintéressant de rapprocher le champ idéologique du champ esthétique, par exemple, pour se donner un découpage opératoire. Dans les deux cas, il y a expérience indéniable quoique complexe et confuse. Dans les deux cas, on distingue, sans trop d'arbitraire, trois niveaux : une conscience travaillée par la norme et le désir, une production d'œuvres et aussi des inflexions, sous forme de résidus irréductibles, traversant des objets voués à une autre fonction. Pour illustrer ce dernier niveau, disons qu'il y a une qualité littéraire évidente dans la Lettre sur les aveugles de Diderot, comme il y a une qualité idéologique au Dictionnaire pbilosophique de Voltaire. La notion du beau n'est pas utile à une esthétique rigoureuse autrement que comme indice spatio-temporel ; la notion de vrai est tenue ici pour aussi peu éclairante dans la constitution d'une théorie de l'idéologie. Le fait que certains discours idéologiques prétendent à l'objectivité référentielle ne change rien à l'affaire. Les jeux mimétiques de la quadratura ou de l'hyper-réalisme n'ont jamais fait de la conformité au référent un critère esthétique fécond, bien au contraire. La perspective d'une "esthétique » du champ idéologique présente donc l'intérêt de surmonter l'opposition simpliste de l'être et de l'apparaître, pour se centrer sur l'être de l'apparaître, et en dégager la logique.

Le second niveau sera traité d'abord. C'est celui des œuvres. Il se prête directement à l'objectivation puisqu'on veut définir un type de discours. À ce stade, il n'est pas question de théorie mais seulement de description. Pour permettre de penser cet objet comme séparable de tout autre, l'étude scientifique doit considérer ce que le discours dit, c'est-à-dire son expressivité, ainsi que les procédés linguistiques par lesquels il le dit, c'est-à-dire sa thétorique et sa stylistique. Les critères de la classification seront donc d'une part thématiques ou sémantiques et d'autre part formels ou syntaxiques. La consistance théorique de la définition est ici proportionnelle à la quantité et à la variété des données recueillies et traitées. 
L'éventail des moyens est aussi large que celui de toute analyse linguistique: règles de validation, grammaire propositionnelle, énoncé illocutoire, discours performatif, etc. Un exercice de décodage original et spirituel a récemment élargi cette typologie dans Le scandale $d u$ corps parlant, Don Juan avec Austin, ou la séduction en deux langues (1980), de Shoshana Felman. Ce ne sont pas là les seules variables constitutives du discours idéologique. Cependant, nous croyons que toute approche qui fait l'économie de ces démarches taxonomiques s'avance dans la gratuité du non-dit sournois et pervers c'est-à-dire de l'invérifiable. La théorie " critique » en a donné un exemple. Le caractère décisoire de la définition de l'objet-idéologie n'entraîne pas sa totale relativité. Ainsi, affirmer que les propositions du "livre blanc" sur la souveraineté-association peuvent et doivent être formellement confrontées avec celles du "livre beige » fédéraliste, sur un autre plan que celui de l'action politique réduite à des intérêts de classe, ce n'est pas opposer une autre définition à la première, c'est ouvrir un registre d'intelligibilité nécessaire à un concept critique encore à venir (sans que la nécessité soit réciproque).

Ce que l'on peut appeler sans abus "l'école de TroisRivières" (UQTR) se situe dans la perspective de cette positivité. On y admet la pluralité des lectures. Depuis 1975, ce groupe a constitué un répertoire des études et un inventaire des traitements visant les idéologies. Quatre directions sont retenues : sémiotique, pragmatique, anthropologique et occultation. Au colloque de l'automne dernier, Claude Savary estimait qu'il se dégage une tendance à dédramatiser le phénomène. Ce retour aux sources brise les exclusives du cercle dogmatique althussérien.

Mais revenons à l'examen de ce qu'Althusser rejette dans la géhenne de la fiction. Le premier niveau, celui de la conscience idéologique, ressortit au registre du vécu. Nous en proposons donc une interprétation anthropologique. Cela soulève évidemment plus de problèmes que la taxonomie des œuvres. Ici, le "versant positif» est pris dans le sens qualitatif où il s'oppose au versant négatif. Quels sont ces problèmes théoriques? On l'a montré, Althusser pratique un 
classement binaire rigide, où tout ce qui n'est pas science est idéologie cependant que l'idéologique est réductible au scientifique. Or il n'y a pas de science du vécu singulier pour les mêmes raisons qu'il n'y a pas de science de la parole, mais seulement une théorie du langage. La cause est entendue, le tragique se trouve évacué. Voilà comment Althusser dépasse le romantisme. On lui accorde que le savant a le choix d'un tel cadrage ; en contrepartie, il renonce à toute inférence métaphysique et laisse à d'autres le soin de parler autrement, sans pouvoir les disqualifier depuis l'intérieur de son discours. Mais un penseur n'est pas placé à cette intersection-là. Il travaille sur les déchets des activités scientifiques autant (ou plus) que sur leurs constructions. Il ne revendique pas pour autant le droit de parler n'importe comment de n'importe quoi, ni celui de s'acharner sur l'indicible. L'enjeu est de taille: c'est l'ancillarité de la philosophie. Althusser a choisi de l'étendre plus loin que Comte ou Wittgenstein, et pourtant ce credo ne va pas de soi. La question reste à débattre. Existe-t-il un lieu d'où je puisse tenir un discours rationnel qui ne coincide ni avec la scientificité, ni avec la rationalisation? S'agit-il vraiment de se faire réducteur ou d'être réduit ? (on pourrait y voir l'enfermement dans le rapport dominant-dominé.). Et d'ailleurs, faut-il exiger que ce soit tout ou rien, " to be or not to be "? Autrement dit, un discours qui prendrait le risque de véhiculer le vécu aurait-il encore du sens (des sens? un sens ?) s'il savait a priori ne pouvoir apporter qu'un degré limité d'élucidation? Je pense qu'il faut répondre affirmativement et placer en exergue du discours philosophique le consentement à l'abandon de la transparence. Que le cogito soit épuisé, il n'y aura que les naifs pour s'en surprendre aujourd'hui, tels les damnés de Dante, impressionnés par l'inscription, à la porte de l'enfer. À l'égard de l'optimisme althussérien, on peut pratiquer "l'ironie socratique, la terrible ironie socratique qui plonge dans l'inconfort et le désespoir ses vis-à-vis " (Ricœur, "Interrogation philosophique et engagement " in Pourquoi la philosophie? 1968). Encore faudrait-il que le vis-à-vis eût des oreilles! Peut-être alors entendrait-il les murmures de ce que ses thèses occultent. Car il n'est pas évident non plus que le lieu scientifique soit une forteresse pure et entièrement insonorisée. Il suffit de lire quinze pages de Képler dans le 
texte pour s'en persuader définitivement et pour se demander comment on lira Althusser dans trois siècles et demi . . .

Comme on prouve le mouvement en marchant, voyons ce que donne à penser une déconstruction anthropologique de l'idéologie. Il n'est nullement besoin pour cela de définir "l'instance homme" comme auto-constituante, ni de la fermer sur l'intentionnel, la facticité, le volontaire ou l'éthique. Les multiples critiques adressées aux anthropologies de la nature humaine ont réglé leur compte à ces facilités. On propose seulement ici de considérer l'humain comme un ordre de fait en mouvement : un vecteur orienté sur une situation concrète, à la fois individuelle et collective. La conscience idéologique est présence au monde, non comme fait accompli mais bien en tant que procès, toujours en alerte. L'intention scientifique (réalité obscure mais elle aussi agissante) a oublié que le lebenswelt n'a pas de sens avant que la conscience n'en fonde un, ou le trouve (les options métaphysiques ne sont d'aucun intérêt ici). De ce point de vue, l'idéologie apparaît comme création lucide et nécessaire, au sens fort du terme. Par la médiation d'un imaginaire mobilisateur et séducteur, elle impose une cohésion au chaos. Elle donne la formulation explicite, totalisante et riche d'une appropriation du monde, affrontée à la durée historique. Cette appropriation n'est pas purement affective : en elle se chevauchent la contemplation et l'action. En effet, pour réussir, son emprise sur le monde doit être autant cognitive que pratique. (Ce sont précisément les deux paramètres que le difficile discours de "Solidarité" essaie de tenir ensemble, au jour le jour, en Pologne. Il lui faut improviser une connaissance de soi-même en situation, et la faire partager à un groupe muet sans compromettre la survivance économique et politique.) La conscience idéologique fournit le moyen de négocier sur le tas avec l'inconnu et le contingent. Par définition, elle suit la démarche concrète et syncrétique du muthos et non la ligne analytique du logos, ce qui n'empêche pas qu'elle ait sa logique régionale, au même titre que le mythe pur, dont Lévi-Strauss isole le système. Par exemple, lorsque l'homme renaissant surmonte sa dispersion mortelle et s'érige en sujet pensant, il se choisit, se reconnaît et se baptise lui-même, tout en rassemblant les hommes dans 
les filets de l'intersubjectivité. Par là, il se donne une tâche autant qu'un nom : il force le passage du chaos au cosmos de la modernité. La conscience idéologique est toujours cosmogonique. Elle s'étudie comme organisation d'une " consonance cognitive" (Festinger, A theory of cognitive Dissonance, 57). L'impensé et l'intérêt y jouent certes un rôle, mais ils interviennent, à des degrés divers, dans toute élaboration symbolique, y compris celle du savoir scientifique, comme Bachelard l'a établi.

Ces quelques indications font voir la maladresse théorique d'Althusser. Poser l'ordre nécessaire du discours scientifique en contrepoids de la cohésion contingente de l'idéologie, comme la théorique transcende l'anecdote, c'est oublier que la vérité scientifique décrit un univers inhabitable, selon l'expression de Dumont. L'idéologie produit la complicité et la familiarité avec un monde ; la science rompt avec le vécu et fragmente ce monde humanisé. Aucun "progrès » ne pourra substituer la seconde à la première. Lorsqu'Althusser reconnaît qu'il faut se résigner à l'éternité de l'idéologie, vu l'inachèvement de l'histoire, nous disons qu'on peut inverser le jugement de valeur : le mouvement historique développe la créativité des sociétés qui s'y trouvent engagées, de gré ou de force. Pour rendre compte des deux objets en équilibrant leur positivité respective, on peut utiliser la distinction entre significance et meaning (Ogden et Richards, 23) qui modernise les catégories épistémologiques d'Aristote. La connaissance apophantique n'est pas exhaustive, affirme le Grec ; à côté d'elle, il reste la connaissance gnomique. Notons que les deux expriment des fonctions cognitives, ce qui nous semble le fondement essentiel à assurer. Bien entendu, nous ne présentons pas ici une contre-théorie de l'idéologie. Il nous suffit d'en avoir fait un objet assez complexe, autonome et encombrant pour justifier qu'on lui accorde un traitement neutre. Les lectures que nous en avons suggérées dans cette première étape se résumeraient ainsi :

1. En tant que champ phénoménal : morphologie, sémantique et syntaxe ; structure à trois niveaux.

2. En tant que parole: psycho-linguistique des fonctions d'instauration et d'appropriation. 
3. En tant que discours : rhétorique ; esthétique ; philosophie du langage (de type anglo-saxon).

4. En tant que conscience: analyse de la fonction pratique ; théorie de la consonance/dissonance cognitive; théorie de la fonction symbolique.

5. En tant qu'impensé : psychanalyse ; épistémologie (au sens strict).

Il reste au moins deux facettes de l'objet à situer : d'une part, l'idéologie comme interprétation de l'événement, ce qui ressortit principalement à l'herméneutique et à la théorie de l'information, et, d'autre part, son statut d'opérateur dans la généralisation et dans la conviction du groupe, ce qui appelle la théorie des communications et la sociologie. Comment marquer ici les bornes du phénomène ? Peut-être en constatant que l'idéologie constitue l'axe dynamique de la représentation collective : ce qui la maintient en vie. Dans la sphère idéologique, en effet, se formulent une pluralité de réponses circonstancielles, adressées à soi et à autrui, devant l'interpellation de l'événement. C'est ce que Sebag appelle «l'urgence du vécu " au secours de laquelle nulle science ne se présente. On entend ici par événement un phénomène discernable dont la probabilité est faible dans un contexte donné. Le contexte, c'est le champ culturel ; l'événement est d'autant plus perturbateur qu'il est moins probable. L'événementiel comporte une échelle de mesure précise. Il apparaît que, dans le système du sens, l'idéologie opère activement le tri et la refonte nécessaires pour faire face, sans éclatement, à l'irruption de l'hétérogène. Première opération: identifier le nouveau en l'apprivoisant pour surmonter l'usure constitutive du temps et rester ainsi en prise sur la situation matérielle, sociale et symbolique. Il y a menace de sombrer dans la fascination de l'altérité. La Méduse ne peut être affrontée de face. Le discours idéologique exemplifie ici l'une des fonctions de la parole en ce qu'il doit muer une figure du tragique, aveugle, muette et inévitable, en une situation dramatique, balisée de responsables, de causes et d'obstacles pondérés, désormais livrés à l'intervention collective. Illusion si l'on veut, mais illusion indépassable : en ce sens, toute idéologie est activiste. Elle canalise une partie de la peur en énergie. Or un certain dix-neuvième siècle allemand et ses héritiers contemporains ne perçoivent que le versant 
dramatique parce qu'ils définissent l'histoire comme un drame. Ce n'est pas le cas des Nietzsche, Scheler, Cassirer et Heidegger qui se sont attachés à l'étude du muthos comme à celle du logos. Cette opération d'assignation du sens se fait généralement par des approximations métaphoriques. S'il s'agissait d'une simple mécanique de récupération truquée, assimilant le même au même par une "fermeture " qu'Althusser oppose à l' " ouverture » de la science, l'idéologie dériverait rapidement vers la névrose obsessionnelle et suicidaire. Or, dès ce premier stade, le même événement suscite des formes idéologiques plurielles, comme autant de sondes ou de paris. Presque toutes échouent. C'est le degré de pertinence objective et subjective par rapport à l'événement qui détermine la légitimité de l'interprétation idéologique. Il est certain que l'autorité des institutions et le rang des locuteurs sont des facteurs qui infléchissent l'expérience subjective de cette pertinence. Les fameux appareils idéologiques d'État (là où il y a État) éclairent cette variable particulière. Seconde opération : l'idéologie effectue sur ce matériau une "mise en intrigue ", pour emprunter hors contexte une expression de Ricœur. L'interprétation est affectée de valeurs positives ou négatives en fonction d'une action collective à entreprendre, à poursuivre et à empêcher. C'est ainsi que l'idéologie la plus conservatrice est forcée de se tourner vers l'altérité pour la reconnaître et la nommer, même quand c'est pour la rejeter. Il se trouve que les intellectuels européens ont une fâcheuse tendance à amputer la sphère idéologique de toutes ces dimensions, pour ne voir que la justification conservatrice ; s'ils ont oublié que l'inauguration précède la justification, c'est qu'ils reflètent de vieilles sociétés encombrées par leurs produits et pauvres en projets. Mais revenons à notre mouton. L'idéologie légitime une action collective. C'est ici qu'apparaît le caractère relationnel de l'objet, lequel ne répond à sa définition que s'il est communiqué. L'efficacité de la communication est mesurable en termes de supports, de circuits et de rapports propositions/dispositions. Ce dernier paramètre est étudié à Trois-Rivières par Claude Panaccio. Les travaux d'Abraham Moles, entre autres, sont du plus haut intérêt en la matière. Les modèles de $\mathrm{Mc}$ Luhan pourraient également en enrichir la lecture (c'est bien le cas de le dire !). 
Une précision d'importance s'impose concernant le tableau qui précède. Les deux opérations présentées séparément ne sont pas successives mais simultanées et investies l'une dans l'autre. L'agent interprète ce qu'il juge communicable et valorise ce qu'il perçoit. Dans l'ordre logique, la séquence semble être la suivante : détection de l'événement, prélèvement, transposition, organisation, généralisation et diffusion. Le résultat se présente comme une configuration complexe où rapports de sens et rapports de forces se trouvent soudés de manière à produire en engagement dont la précarité doit rester partiellement inconsciente. Ce qui est toujours visible, pourtant, c'est le coefficient de désordre inhérent à toute parole idéologique : il tient, d'une part, au chaos dont le contact imprime une trace persistante; d'autre part, il surgit comme irruption de la nouveauté dans l'ordre du discours en place; enfin, la pluralité conflictuelle de cette parole désorganise les réseaux de l'intersubjectivité. Sur ce terrain, il n'y a pas d'autre conscience à lui opposer. J'insiste: ce n'est pas que la fin justifie les moyens ; c'est qu'il n'y a pas d'autre moyen. Que l'idéologie puisse être jugée sur la qualité du projet qu'elle promeut, c'est une question séparée que nous n'abordons pas ici. (Le troisième niveau, celui de l'idéologie-infiltration, peut se penser à partir de là.)

Notre objectif ponctuel consistait à faire valoir les droits philosophiques d'une réflexion anthropologique ouverte. Nous croyons avoir justifié le jugement que nous portions en introduction, à savoir que, dans la théorie althussérienne de l'idéologie, le travail fondationnel et analytique est insuffisant. Résumons. Dans le cadre élargi que nous proposons, le corrélat objectif de l'idéologie, c'est l'événement, plus rigoureux que la notion passe-partout de classe. Lorsqu'elle est vivante, dans le sens où Éliade parle de mythe vivant, l'idéologie maintient le système des représentations ouvert sur la nouveauté. Elle se conçoit donc comme facteur de déséquilibre et d'entropie par rapport à la structure culturelle (que nous examinerons ensuite). On voit mieux en quoi le critère de pertinence est étranger à l'opposition vrai/faux. Cette entropie est à la mesure d'une double disjonction: disjonction de l'homogène pour intégrer l'événement par une prise toujours imparfaite et 
disjonction du groupe en factions concurrentes quant à l'interprétation de l'événement (et non quant à leurs seuls intérêts). L'idéologie manifeste la puissance polysémique des formes symboliques et lorsqu'elle s'affaiblit, la prise d'intelligibilité et de digestion face à l'événement se relâche : c'est le procès de Galilée ou le récent document romain sur l'homosexualité. C'est aussi la défaite du référendum québécois sur la négociation d'un espace d'autonomie: l'instauration d'un statut collectif distinct n'a pas trouvé les formes, les images, les arguments ou les véhicules capables de l'articuler en projet suffisamment pertinent. La position rivale n'y est pas arrivée non plus.

Les premiers à avoir identifié les deux versants (fonctionnel et formel) du champ idéologique furent les sophistes. Ils s'étaient donné une épistémologie constructiviste parce qu'ils percevaient clairement qu'il fallait penser la brèche ouverte par la conscience de l'histoire dans le rempart de la forteresse mythique. C'est pourquoi ils posaient la question des rapports entre le réel et la représentation en termes de création plutôt qu'en termes de vérité/erreur (logique) ou vérité/mensónge (éthique). Une fois le fétichisme métaphysique abandonné, la visée critique peut apercevoir que méconnaître partiellement, c'est encore connaître et peut-être la seule manière de se reconnaître. Car préfigurer, configurer, transfigurer ne se réduisent pas à défigurer. En fin de compte, cù est la figure originelle, sinon dans le faire poétique qui la représente? Au-delà de la tradition, de la norme et de la science, les sophistes ont consciemment formulé une stratégie de la parole de ralliement, sur un terrain où elle est la seule praticable. Ils l'ont désacralisée. Ils ont assumé le tragique de la rupture tel celui qui plante sa tente au-dessus du vide et qui le sait, selon l'image de Henry Miller résumant Rimbaud. Il faudrait donc ménager un espace d'exception pour eux quand on affirme globalement, comme Derrida (congrès de Strasbourg, 1980), que la conception de l'imaginaire comme champ de production autonome n'est pas une question grecque. Le problème de la production d'une vérité consensuelle et sans attaches a été soulevé dans l'Antiquité. Et pour ce faire, la sophistique a dû couper le cordon ombilical entre l'agora et sa source nourricière transcendante. Elle fut violemment rejetée par la cité que son 
discours voulait instituer de droit. Nul autre, avant Machiavel, ne s'engagea dans cette voie étroite que les légistes chinois avaient explorée contre le confucianisme. Le discours des sophistes impliquait donc une transgression sacrilège. Cette problématique inquiétante fut refoulée pour faire place à la vérité triomphante des idées. L'althussérisme refoule, lui aussi, et s'en justifie fort candidement en alléguant des raisons analogues. Il nous confirme par là que la laïcisation de la société est autrement plus complexe que celle de la nature. Nous pensons que son écriture n'y a pas contribué. Et nous préférons Alain lorsqu'il assure que « la philosophie commence avec l'irrespect. »

\section{POUR UNE THÉORIE DE LA FONCTION SYMBOLIQUE}

L'ordre symbolique a ses raisons que la raison dialectique ne connait pas, nous permettions-nous de dire en introduction. Sans nuance péjorative, Lévi-Strauss remarque que la dialectique est une pensée toujours constituante (La pensée sauvage, 1962). Nous pensons que c'est en quoi elle peut travailler efficacement à l'organisation théorique des facettes du champ idéologique, entendu comme axe dynamique de la représentation, en lisant les dissonances et les emboîtements de ses mouvements de dérive entropique. Cependant il y a trois conditions épistémologiques à la scientificité d'une lecture dialectique.

1. La première réside dans l'abandon radical de toute prétention ontologique. Les professions de foi confessant qu'en son fond (double ou triple fond), le réel est dialectique n'apportent qu'infirmité et encombrement dogmatiques.

2. Pour suivre les contours objectifs des contradictions qu'elle vise à articuler, la méthode dialectique doit travailler a posteriori. Car, par elle-même, elle ne construit pas d'objet, comme nous l'avons montré. Il faut donc que soient d'abord menées à leur terme les diverses analyses sectorielles qui appellent cette articulation, et il faut encore que leur positivité soit retenue plutôt qu'engloutie dans cette unité seconde. Marx illustre cet équilibre dans son rapport à la tradition idéaliste qu'il juge plus stimulante que le matérialisme mécaniste, l'un et l'autre étant considérés pour eux-mêmes. 
3. La dialectique, comme toute méthode, doit poser en principe qu'elle apporte une intelligibilité spécifique, limitée et historiquement relativisable. Ce n'est pas là un constat d'échec mais plutôt ce qui reste de Hegel, de Marx et de Engels, une fois leurs interventions respectives sécularisées.

Althusser ne répond à aucune de ces conditions dans son recours à la dialectique. Il faudra attendre que les travaux analytiques de mesure et de vérification, tels ceux de l'école de Trois-Rivières, aient éclairé l'objet-idéologie pour pouvoir mieux saisir le rôle éventuel d'une ou de plusieurs dialectisations du champ idéologique.

Ainsi, non seulement la dichotomie science/idéologie ne peut rendre compte du statut différencié des discours qui composent une problématique, mais surtout elle annule toute possibilité de penser l'autre de l'idéologie ailleurs que dans la pratique théorique. Nous pensons que poser production contre production occulte la question de leur matrice que nous définirons non plus en termes de forces productives mais dans la perspective d'un lieu de procession logique du sens. Car le sens ne génère rien en dehors de lui-même. Il n'a pas d'effets. Il se dissémine en figures dont l'ensemble constitue la fonction symbolique. C'est à propos des rituels pratiqués dans les cas d'accouchement difficile chez les Cuna (rapportés par LéviStrauss) que Sebag délimite ainsi la sphère matricielle: « L'usage de la fonction symbolique se montre ici dans ce qu'il peut avoir d'exemplaire : structuration de contenus hétérogènes, imposition de formes à un donné qui semble d'abord rebelle à tout ordre, instauration d'un langage - c'est-à-dire d'un certain nombre de signifiants majeurs, à travers lesquels une expérience humaine acquiert valeur et permanence. » (Marxisme et structuralisme, 1964). Voilà mis en relation des vecteurs dynamiques et un axe statique, autrement dit l'anthropologique et le formel. Et ce nouveau cadrage nous permet de poser l'hypothèse que l'autre de l'idéologie serait la culture. L'intérêt premier d'une théorie de la fonction symbolique (non exclusive des autres discours) apparaît clairement face au problème qui nous occupe : elle permet l'ancrage du champ idéologique dans un ensemble cohérent où se trouve fondée sa pérennité, par contraste avec la gratuité de la thèse althussérienne sur l'éternité de l'instance idéologique. 
Qu'appelle-t-on exactement le culturel ici ? Précisons d'abord que par culture, on n'entend pas « un contrat passé entre les créateurs et les consommateurs» (Barthes) ni un réseau d'asphyxiantes institutions toutes plus ou moins policières (Dubuffet). Il ne s'agit ni du musée, ni de Paris-Match, encore moins d'une cause idéologique introduite pour distraire les ouvriers de la lutte des classes, comme le suppose Lénine, dans sa correspondance avec Rosa Luxembourg sur le nationalisme polonais, ou encore, comme le pense un Charles Gagnon qui va répétant que la culture est l'opium des petits-bourgeois québécois. Pour simplifier, disons qu'on entend par culture un champ dont le cr.rrélat est la communauté (et non la société). On désigne par là une aire déterminée de communication, régie par un code particulier d'échange des messages linguistiques et non linguistiques. Les messages eux-mêmes se donnent comme éléments observables : leur structure est connue par inférence logique, laquelle en déduit le réseau des formes intégratives qui cimente, sur le plan anthropologique, l'appartenance à la communauté. Nous tenons à distinguer d'emblée ce concept de culture de celui de civilisation. Ce dernier englobe plutôt, à l'horizontale, les élites et les objets policés de plusieurs cultures parentes, à l'échelle de vastes zones géographiques, telle la civilisation méditerranéenne. Élias a montré que le concept de culture s'est forgé pour exprimer la solidarité verticale dans la clôture sur une communauté unique ( $L a$ civilisation des mours, 1939).

Il n'est pas inutile de s'arrêter brièvement à la construction de l'objet-culture pour l'opposer à ce que nous avons dit de l'objet-idéologie. En effet, le contraste est ici frappant entre les sciences de la société et les sciences de la culture. La "chance" épistémologique du phénomène culturel, c'est d'avoir été traité non par des politicologues et sociologues mais bien par des ethnographes, ethnologues et anthropologues non alignés, venant d'horizons aussi dépassionnés que peuvent l'être la géographie, la physique, la linguistique ou l'archéologie. Seuls les apports de l'histoire et de la psychanalyse véhiculaient de véritables controverses. Encore s'agissait-il de débats intellectuels et non d'angoisses salvitiques. On peut situer, en passant, quelques repères concrets. Dans le temps, la 
mise en place se fait, en parallèle avec l'instauration du champ de la psychologie expérimentale allemande. Les deux mouvements sont portés par l'intention critique du positivisme dont ils bénéficient sur le plan méthodologique. C'est avec Tylor (1871) et Boas (1895) que se définit l'interprétation pluraliste des faits culturels. On parle désormais d'aires culturelles plutôt que de $L A$ culture. De mêrne, les écoles anglo-américaines qui s'organisent autour des années vingt, à travers des filiations et des ruptures méthodologiques, invoquent toutes le recours au fait plutôt qu'à la spéculation. C'est le cas des Frazer, Morgan, Mead, Sapir (auquel s'adjoint Barbeau, pendant son enseignement à Ottawa). En France, l'émergence est tardive, sans doute à cause de la répartition universitaire des champs disciplinaires. L'ethnologie doit se dégager d'abord d'une gangue métaphysique imposée par l'horizon philosophique; elle doit ensuite rompre en visière avec les arguties de la «psychologie rationnelle» et de la théorie sociologique, toutes deux enfermées dans l'enseignement de la philosophieomniscience. Les travaux de Lévy-Bruhl en donnent une image exemplaire : l'itinéraire qu'ils empruntent conduit de l'éthique (La morale et la science des mours, 1903) à la possibilité de l'approche scientifique (autocritique des Carnets posthumes, 49). L'obstacle épistémologique résidait dans la notion de "mentalité " où Lévy-Bruhl décèle finalement le décalque de l'âme, sans trop savoir comment le dépasser.

Mais dans tous les cas, l'objet-culture a d'abord été perçu comme le propre d'un autre groupe plutôt que péché originel du chercheur. D'où résistance, altérité et distance. À mesure qu'échouaient les tentatives d'assignation de ces systèmes à l'enfance de nos propres cultures ou à la périphérie d'une nature humaine universelle, l'ethnologue s'est rendu à l'évidence de ce qu'il éprouvait directement : l'irréductibilité de la culturalité. Ce constat met en cause l'unité du système d'intuitions où se meuvent ces autres radicalement autres, telle la certitude de répétition ou de réitération qui gouverne toute weltanschauung archaique, dont la vérification factuelle constante renforce l'évidence. Car l'appréhension de la preuve dépend, elle aussi, de l'appréhension du donné. "Devant une société encore vivante et fidèle à sa tradition, le choc est si fort qu'il déconcerte. " (Tristes tropiques, 1955). C'est l'exotisme de 
l'Inuit, du Montagnais, du Bororo ou du Bambara. Exotisme expérientiel, qui croît en proportion de la fréquentation et dont Victor Segalen nous a laissé d'excellents témoignages. Expérience limite, où l'horizon recule du même pas que la démarche. L'analyse ne peut ni dissoudre ni discréditer la forme des représentations dont elle prend acte. Cette différence ne peut plus être pensée comme manque à combler, ni comme erreur à rectifier (telle que la percevaient les missionnaires européens du $\mathrm{XVII}^{\mathrm{e}}$ en Amérique, dont il reste d'ailleurs quelques survivants défroqués). Une fois l'objet-culture constitué, l'ethnologue n'a rien à entreprendre, sinon de comprendre, - ni rien à changer, sinon lui-même et ses instruments de représentation. Il construit son poste de spectateur marginal, conscient d'être objet d'observation, et ce lieu offre au moins la condition de possibilité d'une scientificité. La «nouvelle ethnologie» des pirates de Parix VII a voulu politiser cette position en dénonçant son parasitisme. Ce mouvement voudrait substituer le dialogue à l'observation : «... ce langage de remplacement qu'on parle pour ne plus entendre le sien (celui de l'observé), pour ne pas découvrir le sien. " dit l'ethno-cinéaste Jean Monod. Cette bonne intention, inverse de celle d'Althusser, ne fait pas de la meilleure science car elle introduit l'illusion d'une autre transparence, concrète celle-là : «... perte de temps en explications pour faire comprendre ce qu'il ne s'agit que d'entendre... « comprendre » emmagasine, «entendre » tend vers ; celui qui comprend dispute, celui qui entend répond. » (« La mécouverte ", in Recherches amérindiennes au Québec X.4, 81). Mais le plus traditionnel des ethnologues se situe plus près de l'étonnement philosophique que de la tranquille assurance althussérienne. Si cette science est inquiète, c'est qu'elle constate l'indice le plus net de la culturalité de son regard. Et ce regard fait d'abord (peut-être exclusivement ?) le tour de la circularité herméneutique par où il se trouve renvoyé à la réflexivité critique. En effet, en raison même de l'exclusion radicale qu'exerce ipso facto la différence d'une intersubjectivité matricielle à une autre, le discours ethnologique est contraint d'interroger ses modalités épistémologiques. C'est ainsi que surgit le déplacement signalé plus haut, à savoir que le souci épistémologique devient partie intégrante de chacune des 
« sciences humaines». Soyons plus précis. Le rationalisme occidental dicte depuis vingt-cinq siècles une grille d'intelligibilité conceptuelle comportant les oppositions binaires universel/singulier, nécessaire/contingent, éternel/transitoire, etc. qui sous-tendent le discours d'Althusser aussi bien que l'humanisme qu'il attaque. Or le corrélat objectif du concept de culture, la communauté, résiste à ces répartitions crispées sur les extrêmes. Pour penser à cette échelle, il faut définir un créneau stable et fini, situé entre le générique et l'individuel. On voit que la déconstruction précède ici la construction. De là - et de là seulement - une rationalité féconde peut assigner un sens à l'apparente gratuité des formes. L'arbitraire irrationnel de la norme se traduit alors en nécessité culturelle rigoureuse, à penser en dehors de toute référence à la fonction. Il est clair que l'instauration originaire de la Loi (au sens de norme) doit être traitée comme une opération axiomatique régissant un territoire logique. La Loi du logos en est une parmi d'autres qui lui sont contemporaines et équivalentes. Ce qui compte, c'est la permanence du code : elle opère l'homogénéisation d'un monde borné dans l'espace et le temps. C'est dans un tout autre sens qu'Althusser parle de changement d'échelle. À notre connaissance, les seuls travaux marxistes français sensibles à ce concept de borne sont les ouvrages conjoints de Lefebvre et Choay sur l'espace urbain et la place que s'y aménagent (ou non) les travailleurs immigrants : ils sont d'autant plus subversifs que leur matière est quotidienne et familière.

Autre trait qui garantit la qualité théorique de l'ethnologie: partout l'ethnographie des compilateurs a précédé le durcissement explicatif. Le nombre, la résistance et la diversité des faits a freiné le délire de systématisation et maintenu ouvert le débat entre les écoles. Un Éliade ou un Lévi-Strauss peuvent élaborer leur modèle sur le fondement d'un large inventaire accumulé avant eux, alors qu'Althusser théorise sur le vide documentaire. La force de cette typologie est dans sa neutralité, c'est-à-dire qu'elle reste remaniable selon les inflexions abstraites nouvelles qu'on veut lui donner.

Quelles sont les constatations du discours ethnologique qui intéressent notre hypothèse sur le culturel en tant qu'autre 
de l'idéologique? La première concerne la vie des formes : il faut la même quantité d'énergie pour conserver que pour innover. Nulle place pour le moralisme dans un tel cadre puisqu'il n'y a rien à défendre. Il y a des faits symboliques à enregistrer et une loi d'organisation à inférer, pour un espace variationnel borné. L'ethnologue reconnaît de droit la différence archétypique. On a renoncé à voir dans la culturalité l'apparaître trompeur d'une vérité universelle sous-jacente dont les cultures seraient les représentations plurielles. Par la médiation des concepts linguistiques, l'inutilité du référent nouménal s'est lentement imposée. Il restait donc des structures symboliques. Ni éternel, ni transitoire, le champ culturel se conçoit comme réseau d'évidences, classification et conservation anonymes des registres symboliques collectifs, structure cohérente de significations renvoyées l'une à l'autre dans un jeu de miroirs. Aucune conscience individuelle ne résume, à elle seule, ce répertoire clos, comme le remarque Dumont, car les figures qu'offre la rotation du kaléidoscope ne peuvent être épuisées par un seul regard. Il nous paraît donc établi que l'anthropologie ne peut suffire à en rendre compte, sous l'angle de la totalité. Le modèle structural reconstitue cette voix dont le vécu n'entend que l'écho. Comme structure, en effet, le culturel sert de frein grammatical là où l'idéologie forge, emprunte ou reçoit sans arrêt des éléments lexicaux nouveaux. À l'état brut, ces données ne sont pas encore des données culturelles, tel l'anglais "switch" avant que le Québécois n'en dérive, fort régulièrement, le verbe du premier groupe «switcher»... De toute manière, l'assimilation (ou l'« indigénation $\gg)$ reste partielle car une aire culturelle n'est pas un lieu étanche. Il est plus exact de concevoir la structure comme gestalt intégrative. Elle garantit, quand elle est saine, cette condition d' " homme rapaillé ", comme dit Miron, sans laquelle nul ne peut prendre le risque d'un projet. L'axe idéologique apparaît comme représentation constituante tandis que l'axe culturel se donne comme représentation constituée, à la fois source et résultante de la première selon des lois de rétroaction complexes et mal connues.

Cette question mérite quelques remarques indicatives. Jusqu'ici, les deux dimensions ont été juxtaposées plutôt qu'articulées. D'un côté, Piaget les pose, comme on le sait, en 
succession chronologique indéfinie mais sans analyser la liaison conceptuelle. Genèse et structure sont pourtant toutes deux simultanément à l'œuvre dans le plus anodin des dialogues. D'un autre côté, Parsons a cherché le substrat commun à la pente structurale de la norme et à la pente fonctionnelle de la valeur. Or, l'entente qu'il a passée avec Kroeber pour répartir les tâches entre le structuralisme et l'anthropologie ressemble à un aveu d'échec (Structure and Process in modern Societies, 60). Enfin, le concept de coupure épistémologique nomme le problème plus qu'il ne le résout. Il nous paraît plus utile de chercher du côté des modèles qui postulent au moins la continuité du matériau. Prenons par exemple les trois fonctions attribuées au système politique chez Lavau. Il s'agit toujours de pouvoir, dans son modèle mais rien n'empêche d'essayer de les transférer dans la vie symbolique. Première fonction : légitimation - stabilisation (communauté, régime, autorités). C'est ce qu'assure la structure culturelle par rapport aux significations.

Seconde fonction: fonction de relève (propositions de changement, critique). Ce serait l'apport idéologique ouvrant sur le nouveau. La troisième, appelée fonction tribunicienne, par Lavau, serait la confrontation entre la règle et les exceptions. La réunion des trois laisse entrevoir ce qui empêche la courbe de se fermer en cercle mort, en la forçant à décrire lentement une spirale. En parallèle, les rapports entre la langue et la parole apportent la clef du potentiel/actuel pour éclairer la liaison. On pourrait dire que la parole conjugue et risque sa proposition personnelle orientée vers l'interlocuteur. Le savant parle à la troisième personne du singulier de l'indicatif; le philosophe au conditionnel et à la première personne du singulier ; enfin l'idéologue, à la première personne du pluriel de l'impératif (plus ou moins explicite). Toutes ces paroles donnent à penser. À un autre niveau, la langue offre le verbe nu, à l'infinitif : c'est l'élément neutre, flottant, intemporel, impersonnel, abstrait. Comme pure forme, l'infinitif porte des possibles et reste disponible. Il est matrice d'un jeu réglé de conjugaison.

À l'échelle d'une communauté, donc, le champ culturel coïncide avec l'équation des règles qui ont toujours déjà 
institué la possibilité de l'intelligibilité concrète et abstraite. Système autorégulateur, toujours conservateur, le champ culturel tend à la fermeture en ramenant l'autre au même pour que cet autre, devenu instrumental, se trouve connecté à l'ensemble. Cette intégration correspond au passage du contenu à la forme. La culture réalise ainsi la clôture et la sédimentation du monde symbolique pour en maintenir la lisibilité. Elle est le foyer qui assure l'homéostasie syntagmatique de la conscience collective et joue dans le sens d'une permanence maximale. Son rôle est conjonctif : d'une part, elle rassemble et classe toutes les significations compatibles autour des deux pôles majeurs que sont l'image de la nature et l'image de l'homme ; d'autre part, elle rassemble les consciences en les investissant du code commun. On voit ici qu'à l'entropie de l'idéologie la culture oppose la néguentropie tautologique nécessaire à toute communication. Car il faut bien qu'il y ait un dictionnaire. Cette nécessité n'est exactement ni théorique ni pratique. Antérieure à toute catégorisation, c'est elle qui positionne le choix occidental de la séparation entre théorie et pratique. C'est elle encore qui règle ce qui est repéré sous le nom d'événement et que nous reconnaissons comme ouverture sur l'innovation tandis que l'archä̈que y reconnaît la figure de son passé à revivre comme autrefois. Encore faut-il ajouter que le repérable renvoyant aux repères, le même donné n'a pas un statut événementiel dans toutes les aires culturelles. Ce qui est général, c'est le fait que l'événement, pour être repéré, doit pouvoir manifester la réactualisation d'un élément recessif de telle structure spécifique.

En amont de toutes les réponses, - et l'idéologie, comme la science, sont des réponses - les formes culturelles ont toujours déjà organisé le découpage des questions. Je le répète, celui qui réduit le cloisonnement des champs culturels à d'insignifiantes variantes de l'inertie folklorique est devenu culturellement insensible par saturation. Il a perdu de vue ce qui permet la vision et qu'une communauté intuitionne confusément dans l'expérience de la dépossession. Être né acadien, cajun, amérindien, québécois ou iranien peut-être, c'est vivre à divers degrés l'expérience d'un déséquilibre tragique: l'expérience innommable du «Speak white» que 
Michèle Lalonde a fait passer du cri à l'incantation. Celle d'une identité collective menacée ; celle du brouillage des arêtes du sens le plus commun; celle de l'ébranlement terrifiant des évidences essentielles à toute coïncidence, à toute perspective, à tout projet commun d'abord, individuel ensuite. Là où l'irruption massive de l'hétérogène n'est plus identifiable, dès lors qu'elle ne se heurte plus à une homogénéité capable de la démarquer, "l'homme agonique " (Miron) devient la proie livrée à la prolifération anarchique d'idéologies délirantes. C'est ce dont Labrousse voulait témoigner. Mais la science la plus correcte, la dramatisation prolétarienne la mieux scénarisée ne peuvent arrêter cette déréalisation irrémédiable du vécu. Dans ce cas, ce qui est atteint, ce sont les coordonnées de définition de la fonction symbolique, ses pôles et ses bornes. L'arbitraire et le chaos submergent alors la culture en dévoilant sa fragilité et sa précarité historique. On peut mesurer divers types et degrés de pathologie culturelle en raffinant le couple genuine culture/sprurious culture que l'on trouve chez Sapir. Mais il se ramènent tous à la figure de l'homme ivre : celui qui ne voit plus parce qu'il voit double. La correction des parallaxes ne vient plus superposer les deux images. Dysfonction symbolique, pourrait-on dire. La parole devient impossible. "Et voilà pourquoi votre fille est périphérique!" ajoutons-nous avec cynisme. Dans ce Babel que de nos jours est un Babel de pacotille télévisée, ce double tragique n'est même plus décelable si ce n'est par quelques poètes.

Il s'est creusé un abîme entre l'althussérisme des années soixante, triomphaliste, logomachique et mécanique, portant dès le départ certains traits de sa décadence, et d'autre part, la difficile prise de parole par une gauche non européenne méfiante, inquiète et interrogative. Englués dans la déculturation, ces intellectuels sont peu nombreux à saisir que la construction d'une idéologie peut être une tâche et, dans certains cas, la plus urgente de toutes, si elle est encore possible. En reprenant à son compte le rationalisme sociologique de Durkheim, Althusser croit avoir fondé une prétendue scientificité d'où il pourrait dissoudre tout maléfice idéologi- 
que. En fait, il nous tient un discours de croyant, avec toutes les violences que cela implique. Comme l'a noté Aron, il n'est pas sûr que la décision politique ait besoin de la dignité philosophique que le discours althussérien cherche à lui donner. La théorie laïque de l'idéologie reste à faire.

Département de philosophie

Collège de Rosemont

\section{BIBLIOGRAPHIE}

ALTHUSSER, Louis, Cours de pbilosophie pour les scientifiques, ronéotypé, 1967, repris et remanié in Philosophie et philosophie spontanée des savants, Maspero, Paris, 1974.

"Idéologie et appareils idéologiques d'État", in La Pensée, no 151 .

Lénine et la philosopbie, Conférence à la Société française de philosophie, le 24 février 1968, Maspero, Paris, 1968.

Lire Le capital, en coll., 2 vol., Maspero, Paris, 1965 et 1968. (c'est cette dernière édition qui est citée.)

trad. et introd. de Manifestes philosophiques de Feuerbach, P.U.F., Paris, 1960.

"Matérialisme historique et matérialisme dialectique ", in

Cabiers marxistes - léninistes no 11.

Pour Marx, Maspero, Paris, 1965.

1964.

"Problèmes étudiants", in La Nouvelle Critique, janvier

Réponse à Jobn Lewis, Maspero, Paris, 1973.

"Sur la dialectique matérialiste ", in La pensée, août 63.

"Sur la révolution culturelle", in Cabiers marxistes léninistes, nos 13-14.

LAGUEUX, Maurice, «L'usage abusif du rapport science/idéologie 》 in Culture et langage, collectif, HMH, Montréal, 1973.

SAVARY, Claude, "Matérialisme bistorique et science de l'bomme" in Proceedings of the XVtb World Congress of Philosophy, Sofia, 1974.

DUMONT, Fernand, Les idéologies, P.U.F., Paris, 1974.

KARSZ, Saül, Théorie et politique : Louis Altbusser, Fayard, Paris, 1974. Les passages cités se trouvent p. 18.

ARON, Raymond, D'une sainte famille à l'autre, Gallimard, Paris, 1969.

RANCIÈRE, Jacques, La leçon d'Altbusser, Gallimard, Paris, 1974.

BADIOU, Alain, "Le (re)commencement du matérialisme dialectique » in Critique, no 240 . 
POULANTZAS, Nicos, Pouvoir politique et classes sociales, Maspero, Paris, 1970.

Contre Althusser, collectif, U.G.E., Paris, 1974.

LABROUSSE, Bernard, De l'idéologie dominée, Nouvelle optique, Montréal, 1978.

SEBAG, Lucien, Marxisme et structuralisme, Petite bibliothèque Payot, Paris, 1964.

DUFOUR, Michel, «Lacan et Althusser: une rencontre et des promesses » in Culture et Langage, collectif, HMH, Montréal, 1973.

LAURIN-FRENETTE, Nicole, Production de l'État et formes de la nation, Nouvelle optique, Montréal, 1978.

ARON, Raymond, La lutte de classes, Gallimard, Paris, 1.964.

AUZIAS, Jean-Marie, Clefs pour le structuralisme, Seghers, Paris, 1967.

ELLUL, Jacques, L'illusion politique, Laffont, Paris, 1965.

ARENDT, Hannah, La condition de l'bomme moderne, trad. Fradier, Calmann - Lévy, Paris, 1961.

DUFRENNE, Mikel, Pour l'bomme, Seuil, Paris, 1968.

Esprit, no 350. Les structuralismes

FESTINGER, Leon, A Theory of cognitive dissonance, Row \& Peterson, Evanston, Ill, , 1957.

OGDEN, C.K., RICHARDS, I.A., The Meaning of Meaning, Harcourt, Brace and Company, New York, 1923. 